\title{
Infalling Streamer-Disk-Outflow System in a Nearby High-mass Star Formation Region
}

Xi Chen ( $\nabla$ chenxi@gzhu.edu.cn )

Guangzhou University https://orcid.org/0000-0002-5435-925X

\section{Zhiyuan Ren}

National Astronomical Observatories, CAS

\section{Da-Lei Li}

Xinjiang Astronomical Observatory, Chinese Academy of Sciences

Tie Liu

Shanghai Astronomical Observatory

Ke Wang

Kavli Institute for Astronomy and Astrophysics, Peking University

\section{Zhi-Qiang Shen}

Shanghai Astronomical Observatory

\section{Simon Ellingsen}

University of Tasmania https://orcid.org/0000-0002-1363-5457

\section{Andrey Sobolev}

Ural Federal University https://orcid.org/0000-0001-7575-5254

\section{Ying Mei}

Guangzhou University

\section{JingJing Li}

Purple Mountain Observatory, Chinese Academy of Sciences

\section{Yue-Fang Wu}

Kavli Institute for Astronomy and Astrophysics, Peking University

\section{Kee-Tae Kim}

Korea Astronomy and Space Science Institute

\section{Article}

Keywords: high mass star, theoretical models, numerical simulations

Posted Date: April 29th, 2021

DOI: https://doi.org/10.21203/rs.3.rs-402900/v1 
License: (c) (i) This work is licensed under a Creative Commons Attribution 4.0 International License. Read Full License 


\title{
LETTER
}

\section{Infalling Streamer-Disk-Outflow System in a Nearby High-mass Star Formation Region}

\author{
Xi Chen ${ }^{1,2,3 *}$, Zhi-Yuan Ren ${ }^{4}$, Da-Lei Li ${ }^{5}$, Tie Liu $^{2,3}$, Ke Wang ${ }^{6}$, Zhi-Qiang Shen ${ }^{2,3}$, Simon P. Ellingsen ${ }^{7}$, Andrej M. Sobolev ${ }^{8}$,
} Ying $\mathrm{Mei}^{1}$, Jing-Jing $\mathrm{Li}^{9}$, Yue-Fang $\mathrm{Wu}^{6}$, and Kee-Tae Kim ${ }^{10,11}$

Theoretical models and numerical simulations suggest that high mass star (with mass $>8 M_{\odot}$ ) can be formed either via monolithic collapse of a massive core ${ }^{1,2}$ or competitive accretion ${ }^{3,4}$, but the dominant mechanism is currently unclear ${ }^{5,6}$. Although recent high resolution observations with the Atacama Large Millimeter/submillimeter Array (ALMA) have detected physical and kinematic features, such as disks ${ }^{7-9}$, outflows ${ }^{10,11}$ and filamentary structures $^{12,13}$ surrounding the high mass young stellar objects (HMYSO), direct detection of the infalling gas towards the HMYSO is still the key to distinguish the different scenarios. Chemically fresh gas inflows have been detected towards low-mass stars ${ }^{14}$ being formed, which are consistent with the accretion-disk-outflow process $^{15}$. In this work we report the detection of a chemically fresh inflow which is feeding HMYSO growth in the nearby highmass star-forming region G352.63-1.07. High quality images of the dust and molecular lines from both ALMA and the Submillimeter Array (SMA) have consistently revealed a gravitationallycontrolled gas inflow towards a rotating structure (disk or torus) around the HMYSO. The HMYSO is also observed to have an outflow, but it can be clearly separated from the inflow. These kinematic features provide observational evidence to support the conjecture that high-mass stars can be formed in a similar process to that observed in the low-mass counterparts. The chemically fresh infalling streamers could also be related with the disk configuration, fragmentation and accretion bursts that occur in both simulations and observations.

Research into the process of high-mass star formation is impeded by a number of factors. Specifically, the nearest samples of high-mass star formation are significantly more distant than those for low-mass star formation, and that high-mass stars are often formed in clustered environment, whereas the low-mass stars are more usually formed in isolation. More isolated high-mass star-forming regions at relatively small distances $(<1 \mathrm{kpc})$ are therefore rare and favorable targets to reveal the critical processes in forming high-mass stars. Trigonometric

${ }^{1}$ Center for Astrophysics, GuangZhou University, Guangzhou 510006, China; chenxi@gzhu.edu.cn ${ }^{2}$ Shanghai Astronomical Observatory, Chinese Academy of Sciences, 80 Nandan Road, Shanghai, 200030, China

${ }^{3}$ Key Laboratory of Radio Astronomy, Chinese Academy of Sciences, China

${ }^{4}$ National Astronomical Observatories, Chinese Academy of Sciences, Datun Rd. A20, Beijing, China

${ }^{5}$ Xinjiang Astronomical Observatory, Chinese Academy of Sciences, 830011 Urumqi, Chin

${ }^{6}$ Kavli Institute for Astronomy and Astrophysics, Peking University, 5 Yiheyuan Road, Haidian

District, Beiing 100871 Chin

${ }^{7}$ School of Natural Sciences, University of Tasmania, Private Bag 37, Hobart, Tasmania 7001, Australia

Ural Federal University, 19 Mira street, 620002 Ekaterinburg, Russia

Purple Mountain Observatory, Chinese Academy of Sciences, Nanjing 210008, China

${ }^{10}$ Korea Astronomy and Space Science Institute, 776 Daedeokdae-ro, Yuseong-gu, Daejeon 34055, Republic of Korea

${ }^{11}$ University of Science and Technology, Korea (UST), 217 Gajeong-ro, Yuseong-gu, Daejeon 34113 , Republic of Korea parallax observations of the $6.7 \mathrm{GHz}$ methanol masers in the G352.631.07 star formation region measured a distance of $0.69 \mathrm{kpc}$, which locates it in an inter-arm region ${ }^{16}$, wherein the gas environment could be much less clustered than the Galactic-arm regions. These properties make G352.63-1.07 rightly the expected target to investigate the detailed physical and kinematic features.

Figure 1 shows the ALMA 3 mm and SMA $1.1 \mathrm{~mm}$ dust continuum emissions (see Method), which have a spatial resolution of $\sim 0.01 \mathrm{pc}$ and should closely trace the dense-core gas. Their contours are overlaid on the 2MASS $2 \mu \mathrm{m}$ Ks and three-color GLIMPSE IRAC images. Five dust emission components can be clearly identified, labelled as M1 to M5. They should represent individual high-mass dense cores. M1 is the most compact and strongest one therein. It is also associated with strong $6.7 \mathrm{GHz}$ methanol maser emission ${ }^{16}$, which is supposed to be exclusively associated with HMYSOs ${ }^{17}$. The 2 MASS $2 \mu \mathrm{m}$ image around M1 (see Figure 1) exhibits a bright bipolar emission feature with a roughly symmetrical distribution over M1. The intense 2MASS $2 \mu \mathrm{m}$ emission usually traces the shock-excited molecular $\mathrm{H}_{2}$ and the emission feature also has a comparable morphology with the shocked outflow in IRAS 20126+4104 (ref. 18). As another well known shock tracer, the IRAC $4.5 \mu \mathrm{m}$ emission also shows a largely overlapped spatial extent with the $2 \mu \mathrm{m}$ emission. The two emission features thus both strongly indicate a bipolar outflow from M1 (see Figure 1). In contrast to the shock emissions, the dense and warm gas tracers (e.g. $\mathrm{CH}_{3} \mathrm{CN}$ and $\mathrm{CH}_{3} \mathrm{OH}$ transitions) are only detected toward $\mathrm{M} 1$, suggesting that warm compact gas is only associated with $\mathrm{M} 1$. The $\mathrm{CH}_{3} \mathrm{CN}$ transitions provide a gas temperature estimate of $\sim 170 \mathrm{~K}$ (see Method). M1 thus should be a compact hot molecular core driving a bipolar outflow.

The bipolar outflow is also clearly detected in the molecular transitions (see Figure 2). The ALMA $\mathrm{HCO}^{+}$and SMA CO lines all exhibit high-velocity emission wings on their spectra (Figure 2 right panels) with a speed up to $\sim 15 \mathrm{~km} \mathrm{~s}^{-1}$ with respect to light of sight (LOS). The emission regions of these high-velocity components are closely overlapped with the infrared shock emissions, and also have a symmetric distribution with respect to M1. As denoted with dashed lines, this bipolar-outflow has a large opening angle $\left(\sim 60^{\circ}\right)$. Considering its spatial separation of $\sim 20000$ au (or $0.1 \mathrm{pc}$ ) from the driving source, and assuming an inclination angle of $60^{\circ}$ with respect to LOS (same as the angle of inclination of the disk/torus rotational axis; see below), it then implies a dynamic ages of $\sim 3600$ years. With this time scale, the mass-loss rate for the M1 outflow is estimated to be $1.4 \times 10^{-5} \mathrm{M}_{\odot}$ year $^{-1}$ (see Method).

On the other hand, although the molecular outflow lobes and infrared shock emissions have similar spatial extents, the lobes actually have clumpy structures and are less dispersed than the infrared emission. As an explanation, the outflow components ejected from M1 may not be entirely interacting with the surrounding gas. The infrared emission at $2 \mu \mathrm{m}$ and $4.5 \mu \mathrm{m}$ would trace considerably more energetic 
fraction of the outflow that is sufficient to generate shocks. The less intense molecular outflow could propagate to a larger distance, particularly as indicated by the southwestern $\mathrm{HCO}^{+}$lobes (Figure 2 upper panel), which have a projected distance of $0.1 \mathrm{pc}$ from $\mathrm{M} 1$.

The SMA images of the molecules DCN, $\mathrm{DCO}^{+}$and $\mathrm{H}_{2} \mathrm{CO}$ show that these three molecular lines are emitting from a linear structure aligned with M1, M2 and M3 at a position angle of $\sim 165^{\circ}$ (see main panel of Figure 3). The DCN and $\mathrm{H}_{2} \mathrm{CO}$ emission is mostly located in the region between $\mathrm{M} 2$ and $\mathrm{M} 3$ and peaks at $\mathrm{M} 1$, but $\mathrm{DCO}^{+}$is only observed in the $\mathrm{M} 2$ and $\mathrm{M} 3$ regions. Their position-velocity $(\mathrm{P}-\mathrm{V})$ profiles along the direction of M1-M2-M3 are shown in the lower panels of Figure 3. The velocity field of the blue- and red-shifted sides both exhibit an symmetric increasing feature towards the center, which is similar to that seen in other Keplerian-rotating disks ${ }^{19}$. It suggests the presence of a rotating structure associated with M1. The enclosed mass calculated by fitting a Keplerian rotation model to the $\mathrm{P}-\mathrm{V}$ profiles is $9.0 / \sin ^{2} \theta \mathbf{M}_{\odot}$ (see Method), where $\theta$ is the angle of inclination of the disk/torus rotational axis with respect to the LOS. Combining the structures toward the disk/torus edge at $\mathrm{M} 3$ traced by $\mathrm{DCO}^{+}$and dust emission, an inclination angle $\theta$ of $\sim 60^{\circ}$ is inferred, indicating an enclosed mass of $\sim 12 \mathrm{M}_{\odot}$ in system. This mass is consistent with that estimated from the $1.1 \mathrm{~mm}$ dust emission detected with the SMA (see Method).

The molecular emissions show the rotational system to have a radius of $\sim 10^{\prime \prime}(7000 \mathrm{au})$, which is larger than the typical size of a HMYSO revealed by ALMA observations $(\sim 1000 \text { au })^{7-9,20,21}$. Instead, the rotating gas has a larger spatial size, a less regular shape, but is still flattened to some degree onto the mid-plane of the rotational axis. It could be rotating torus surrounding the inner disk, which has a radius of $<1^{\prime \prime}$ (or $<700 \mathrm{au}$ ). There is also a smooth temperature increasing trend towards the center as inferred from the $\mathrm{H}_{2} \mathrm{CO}$ transitions (see Method for details). This also suggests the rotating torus to be the surrounding structure closely associated with the inner HMYSO. A similar circumstellar torus with comparable radius (8000-12000 AU) was detected around the clump SMA1 in S255N (ref. 22).

The hypothesis of rotating torus with decreasing temperature is also supported by the $\mathrm{DCO}^{+}$emission, which only detected towards the outer edge of the rotational system, but absent in the inner region. This molecule is found to trace coldest disk locations ${ }^{23,24}$ and the outer disk ring in a low-mass protostar ${ }^{25}$. Taken together, we suggest that the condensations of $\mathrm{DCO}^{+}$close to the M2 and M3 dust emission reflects higher material accumulation at those locations due to an edgebrightening effect along the LOS, or perhaps a thickened ring at the edge (similar to a 'doughnut'). The orientation of the rotational system perpendicular to the bipolar-outflow driven by M1 (see Figure 2), also supports the hypothesis that it is a rotational disk or torus.

It should be noted that the centre of the rotating system is not coincident with the peak of M1 emission, but with an offset of of $\sim 1000$ au (or $\sim-1.5^{\prime \prime}$; see lower panel of Figure 3 ) from the position of the M1 peak along the disk/torus axis. The position of the center of gravitation is likely to coincide with the position of the YSO and displacement with respect to the dust emission peak may be caused by inhomogeneous material distribution in the disk/torus system. The differences in the distributions of DCN (detected primarily toward the northwest) and $\mathrm{H}_{2} \mathrm{CO}$ (detected primarily toward the southeast) also suggests different gas density and temperature environments, as well as chemical diversity over the disk/torus structure.

As shown in Figure 4, the ALMA $\mathrm{HC}_{3} \mathrm{~N}$ and $\mathrm{CCH}$ emissions exhibit an overall north-south oriented filament with a scale of $\sim 30^{\prime \prime}(0.1$ pc, see upper panel of Figure 4). The two species both have a noticeable velocity variation around the M1 position, which may indicate a more extended gas motion, such as an accretion streamer towards M1 The $\mathrm{HC}_{3} \mathrm{~N}$ and $\mathrm{CCH}$ are both chemically early-type molecules ${ }^{29}$, thus are expected to trace colder gas regions. The gas temperature derived from our observations of two $\mathrm{CCH}$ hyperfine transitions is typically $\sim 10 \mathrm{~K}$ (see Method), which is consistent with that measured in other sources $^{23,30}$. These species are likely to be depleted in more evolved dense cores, resulting in less close correspondence between the molecular emission from $\mathrm{HC}_{3} \mathrm{~N}$ and $\mathrm{CCH}$ and the dust continuum emission. It is noted that another streamer with distinct velocities are also seen along M4 and M5. It could be driven by the outflow from M4 and eventually enters the main filament at the M5 position.

We inspected the different molecular emissions closer to M1 and found streamers along two more directions traced by the chemically fresh molecues $\mathrm{HC}_{3} \mathrm{~N}, \mathrm{CCH}$ and $\mathrm{HCO}^{+}$, as shown in Figure 4 (middle and lower panels). As shown in middle panels, the ALMA $\mathrm{HCO}^{+} \mathrm{e}-$ mission exhibits a noticeable spatial extension from southwest to northeast over M1, denoted as PV2 direction. The PV diagram along this direction exhibits velocity gradient with increasing blueshift towards M1. The SMA HCO ${ }^{+}$emission is less extended than its ALMA counterpart, which is possibly due to the less complete spatial coverage of the SMA baselines. But it shows an overall consistent velocity gradient on the PV diagram. Such velocity increasing feature toward M1 is different from either outflow or core rotation. Moreover, the $\mathrm{HCO}^{+}$ extension is almost perpendicular to the mid-plane of the rotating torus. The $\mathrm{HCO}^{+}$feature is therefore more likely to indicate an infall motion towards the mid-plane.

As shown in lower panels, the ALMA $\mathrm{HC}_{3} \mathrm{~N}$ and $\mathrm{CCH}$ emissions both exhibit another extension towards northeast as indicated by PV3 direction. And their PV diagrams again exhibit a steep but smooth gradient towards the mid-plane with a velocity change of $V-V_{\text {sys }}=0$ to $-4 \mathrm{~km} \mathrm{~s}^{-1}$ over the range $r=20^{\prime \prime}$ to $0^{\prime \prime}$, The corresponding velocity gradient is approximately $60 \mathrm{~km} \mathrm{~s}^{-1} \mathrm{pc}^{-1}$, which is comparable with that seen in Orion ${ }^{31}$.

Assuming that the streamers are free falling due to the gravity of a central mass, we can fit the P-V diagrams to a very consistent degree by adjusting the inclination angle of the streamer, and the projection height of the landing point of the streamer onto the disk/torus. The best-fit central mass $\left(\sim 12 M_{\odot}\right)$ is also very consistent with the value derived from the rotational disk/torus system (see Method for details).

The P-V distributions for the chemically fresh molecular species show gravitationally-controlled infalling streamers feeding material onto the high-mass protostar embedded in M1 from at least two directions. The two streamers are also likely to have different landing points. But the landing points are both located on the mid-plane of the rotating structure (torus). The landing points appear to be offset by $\sim 3000 \mathrm{au}$ from the central protostar. We estimate the typical free-fall timescale for these streamers. They are estimated to have $t_{f f} \sim 4.6 \times 10^{4}$ years. The total infall rate of material from these streamers accordingly is $\sim 1 \times 10^{-4} \mathrm{M}_{\odot}$ year $^{-1}$ (see Method for details). This implies that the infalling streamers can contribute up to $40 \%$ of accreting material in the accretion process see Method).

Numerical simulations predict that asymmetric accretion flows could have a more significant impact on the accretion rate of a protostar compared axisymmetric infalling-envelope $e^{26-28}$. Direct imaging of such structures is thus crucial but still rare so far. The presence of chemically fresh accretion streamers has been detected on scales of 10000 au towards a low-mass star forming region in recent ALMA observations of carbon-chain molecular species ${ }^{14}$. There are no confirmed previous observations of this kind of an accretion flow in a high-mass star formation region, although filament or hub structures have been detected in a number of regions ${ }^{12,13}$.

Our results show clear signatures of infalling streamers towards a rotating disk/torus system around the HMYSO. And the streamers should mainly consist cold dense gas due to their detection in the chemically fresh molecules. The HMYSO is in a sparsely clustered en- 
vironment and also associated with a set of bipolar-outflows. But the other gas components can all be clearly distinguished from the infalling flows. These observations support the hypothesis that high-mass stars can efficiently form via a monolithic accretion provided that infalling material can be transported into the center in an efficient process. The chemically fresh infalling streamers detected here are also similar to those observed in low-mass star formation, suggesting that they play an important role in both low- and high-mass star formation.

It is also noted that the streamers are falling onto the rotating structure instead of directly onto the central star. The infall in this manner may support the disk accretion with a continuous mass supply, and eventually more efficiently transfer the mass onto the star. But on the other hand, the impact from the infalling gas could also cause a disk fragmentation, generate instabilities, including accretion burst$\mathrm{s}$ or episode accretion phenomena, as has been detected in a number of HMYSOs (ref. 32 and references therein). New numerical calculations are required to investigate such kinematic processes and similar high angular resolution observations in additional regions are required to clarify the frequency and importance of these streamers during the high-mass star formation process.

1. McKee, C. F. \& Tan, J. C. Massive star formation in 100,000 years from turbulent and pressurized molecular clouds. Nature 416, 59-61 (2002).

2. McKee, C. F. \& Tan, J. C. The formation of massive stars from turbulent cores. Astrophys. J. 585, 850-871 (2003).

3. Bonnell, I. A., Vine, S. G., Bate, M. R. Massive star formation: nurture, not nature. Mon. Not. R. Astron. Soc. 349, 735-741 (2004).

4. Bonnell, I. A. \& Bate, M. R. Star formation through gravitational collapse and competitive accretion. Mon. Not. R. Astron. Soc. 370, 488-494 (2006).

5. Zinnecker, H. \& Yorke, H. W. Toward understanding massive star formation. Annu. Rev. Astron. Astrophys. 45, 481-563 (2007).

6. Tan, J. C. et al. Massive star formation. Protostars and Planets VI 149C172 (2014)

7. Johnston, K. G. et al. A Keplerian-like disk around the forming O-type star AFGL 4176. Astrophys. J. Lett. 813, L19 (2015).

8. Ilee, J. D. G11.92-0.61 MM1: a Keplerian disc around a massive young proto-O star. Mon. Not. R. Astron. Soc. 462, 4386-4401 (2016).

9. Zapata, L. A. et al. An asymmetric keplerian disk surrounding the O-type protostar IRAS 16547-4247. Astrophys. J. 872, 176 (2019).

10. Zinchenko, I. I., Liu, S.-Y., Su, Y.-N., Wang, K.-S. \& Wang, Y. Dense Cores, Filaments, and Outflows in the S255IR Region of High-mass Star Formation. Astrophys. J. 889, 43 (2020).

11. Goddi, C., Ginsburg, A., Maud, L. T., Zhang, Q. \& Zapata, Luis A. Multidirectional mass accretion and collimated outflows on scales of 100-2000 au in early stages of high-mass protostars. Astrophys. J. 905, 25 (2020).

12. Hacar, A., Tafalla, M., Forbrich, J., Alves, J., Meingast, S., Grossschedl, J. \& Teixeira, P. S. An ALMA study of the Orion Integral Filament. I. Evidence for narrow fibers in a massive cloud. Astron. Astrophys. 610, 77 (2018).

13. Chen, H.-R. et al. Filamentary accretion flows in the Infrared dark cloud G14.225-0.506 revealed by ALMA. Astrophys. J. 875, 24 (2019).

14. Pineda, J. E., Segura-Cox, D., Caselli, P., Cunningham, N., Zhao, B., Schmiedeke, A. Maureira, M. J. \& Neri, R. A protostellar system fed by a streamer of 10,500 au length. Nat. Astron. 4, 1158-1163 (2020).

15. Shu, F. H., Adams, F. C. \& Lizano, S. Star formation in molecular clouds: observation and theory. Annu. Rev. Astron. Astrophys. 25, $23-81$ (1987).

16. Chen, $X$. et al. High-mass star formation in the nearby region G352.6301.067. I. Parallax. Astrophys. J. 871, 198 (2019).

17. Xu, Y., Li, J. J., Hachisuka, K., Pandian, J. D., Menten, K. M. \& Henkel, C. A high-sensitivity $6.7 \mathrm{GHz}$ methanol maser survey toward $\mathrm{H}_{2} \mathrm{O}$ sources. Astron. Astrophys. 485, 729 (2008).

18. Cesaroni, R. et al. A close-up view of a bipolar jet: Sub-arcsecond nearinfrared imaging of the high-mass protostar IRAS 20126+4104. Astron Astrophys. 549, 146 (2013).

19. Beltrán, M. T. et al. Filamentary structure and Keplerian rotation in the high-mass star-forming region G35.03+0.35 imaged with ALMA. Astron Astrophys. 571, 52 (2014).

20. Maud, L. T. et al. Chasing discs around O-type (proto)stars. ALMA evidence for an SiO disc and disc wind from G17.64+0.16. Astron. Astrophys. 620, 31 (2018).
21. Maud, L. T. et al. Substructures in the Keplerian disc around the O-type (proto-)star G17.64+0.16. Astron. Astrophys. 627, L6 (2019).

22. Zemlyanukha, P. M., Zinchenko, I. I., Salii, S. V., Ryabukhina, O. L. \& Liu, S. -Y. The spatial-kinematic structure of the region of massive star formation S255N on various scales. Astronomy Reports. 62, 326-345 (2018).

23. Ginard, D. et al. Spectral line survey of the ultracompact HII region Monoceros R2. Astron. Astrophys. 543, 27 (2012).

24. Pety, J., Goicoechea, J. R., Hily-Blant, P., Gerin, M., Teyssier, D. Astron. Astrophys. 464, L41-L44 (2007).

25. Öberg, Karin I. et al. Double $\mathrm{DCO}^{+}$rings reveal $\mathrm{CO}$ ice desorption in the outer disk around IM Lup. Astrophys. J. 810, 112 (2015)

26. Kuznetsova, A., Hartmann, L. \& Heitsch, F. The origins of protostellar core angular momenta. Astrophys. J. 876, 33 (2019).

27. Kuffmeier, M., Calcutt, H. \& Kristensen, L. E. The bridge: a transient phenomenon of forming stellar multiples. Sequential formation of stellar companions in filaments around young protostars. Astron. Astrophys. 628, 112 (2019).

28. Wurster, J., Bate, M. R. \& Price, D. J. There is no magnetic braking catastrophe: low-mass star cluster and protostellar disc formation with non-ideal magnetohydrodynamics. Mon. Not. R. Astron. Soc. 489, 17191741 (2019)

29. Bergin, E. A. \& Tafalla, M. Cold dark clouds: the initial conditions for star formation. Annu. Rev. Astron. Astrophys. 45, 339-396 (2007).

30. Sanhueza, P. et al. Distinct chemical regions in the "Prestellar" infrared dark cloud G028.23-00.19. Astrophys. J. 773, 123 (2013).

31. Hacar, A., Alves, J., Tafalla, M., \& Goicoechea, J. R. Gravitational collapse of the OMC-1 region. Astron. Astrophys. 602, L2 (2017).

32. Chen, $X$. et al. New maser species tracing spiral-arm accretion flows in a high-mass young stellar object. Nat. Astron. 4, 1170-1176 (2020).

Materials \& Correspondence Correspondence and requests for materials should be addressed to X. C. (email: chenxi@gzhu.edu.cn).

Acknowledgements X.C. was supported by the National Natural Science Foundation of China (11590781 and 11873002), Project Supported by Guangdong Province Universities and Colleges Pearl River Scholar Funded Scheme (2019). S.P.E. acknowledges the support of ARC Discovery Project (project number DP180101061).

Author Contributions X.C. and Z.Y.R. wrote the initial manuscript, obtained and reduced the data, and led the initial SMA observing proposals. D.L.L. and Y.M. made the gas temperature calculations. T.L. and K.W. were involved in the initial ALMA observing proposal, reduced the ALMA data. Z.Q.S., S.E. A.S., J.J.L. and Y.W. helped to improve the manuscript. S.Y.L., N.J.E. and K.T.K. were involved in the initial ALMA observing proposal.

Author Information The authors declare no competing financial interests. Readers are welcome to comment on the online version of the paper. 


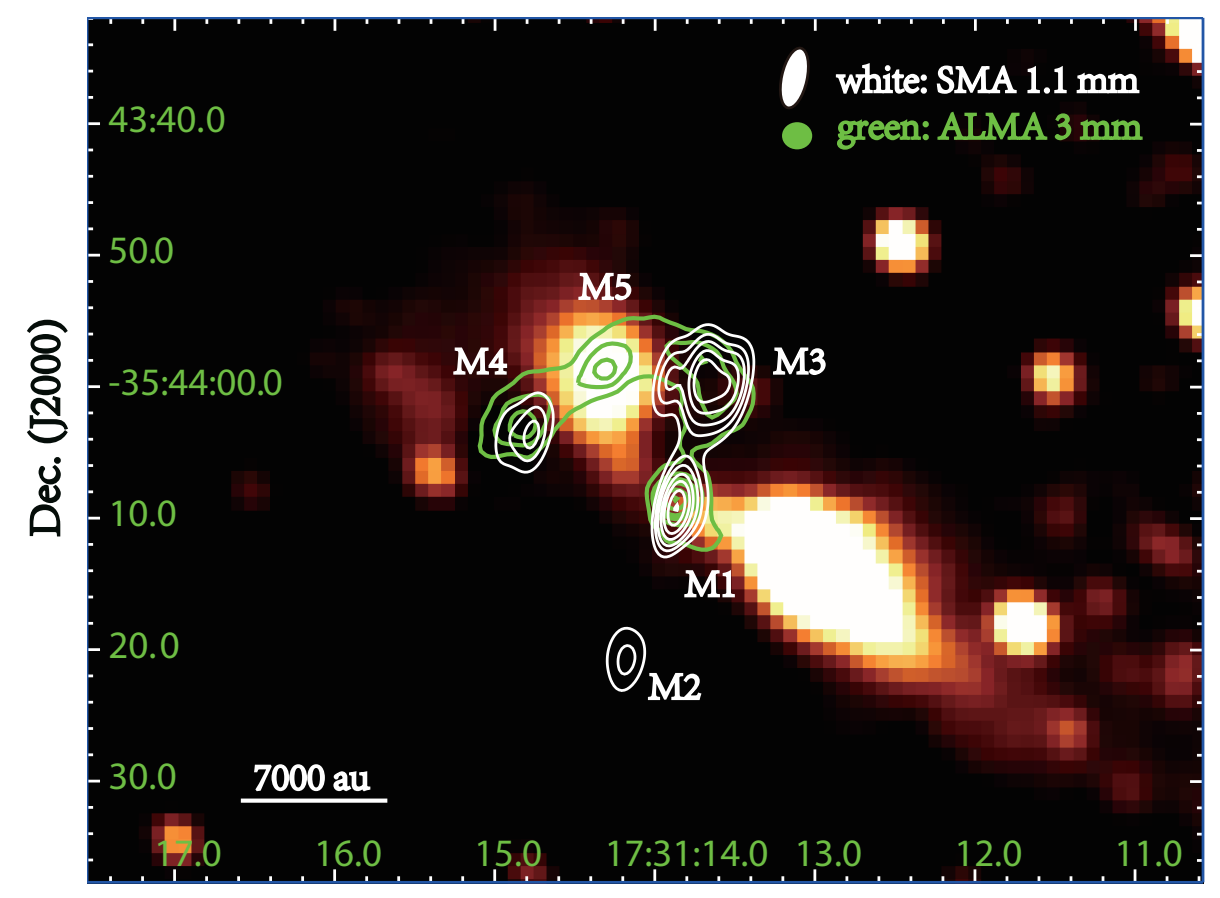

\section{R. A. (J2000)}

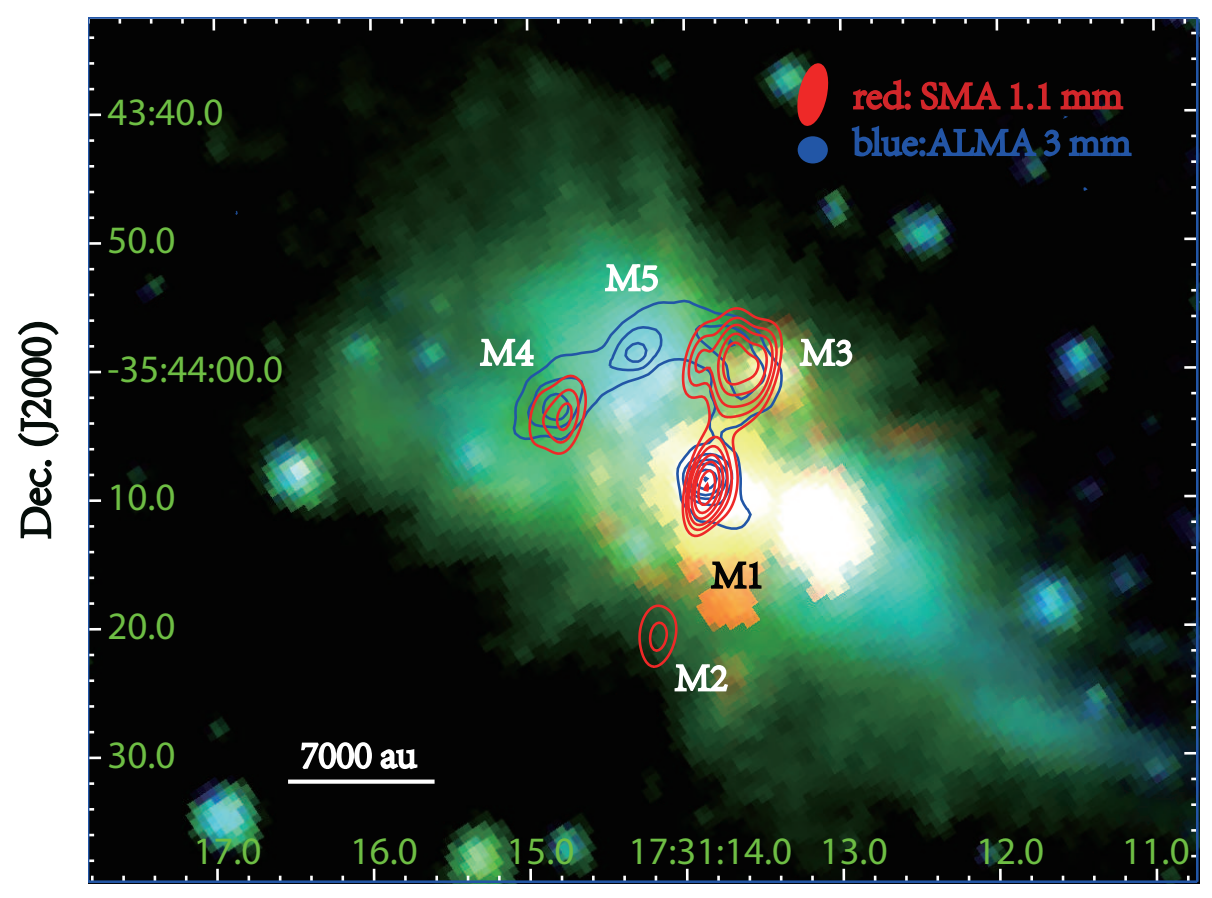

\section{R. A. (J2000)}

Figure 1: Millimeter dust emission maps imaged with ALMA and SMA towards G352.63-1.07. Green and blue contours (in upper and lower panels, respectively) show the $3 \mathrm{~mm}$ dust emission detected with ALMA, with contour levels of $(1,2,3,4,6,8) \times 1 \mathrm{mJy} \mathrm{beam}^{-1}$. White and red contours (in upper and lower panels, respectively) show the $1.1 \mathrm{~mm}$ dust emission detected with SMA, with contour levels of (3, 5, 7, 10, $13,16,19) \times 4$ mJy beam $^{-1}$. The corresponding dust emission components are marked with M1-M5. The beam shapes of the two observations are given by the ellipses. The background are the near-infrared 2MASS Ks $2 \mu \mathrm{m}$ for upper panel, and the three-color GLIMPSE IRAC image composite with $8.0 \mu \mathrm{m}$ in red, $4.5 \mu \mathrm{m}$ in green, and $3.6 \mu \mathrm{m}$ in blue for lower panel. 

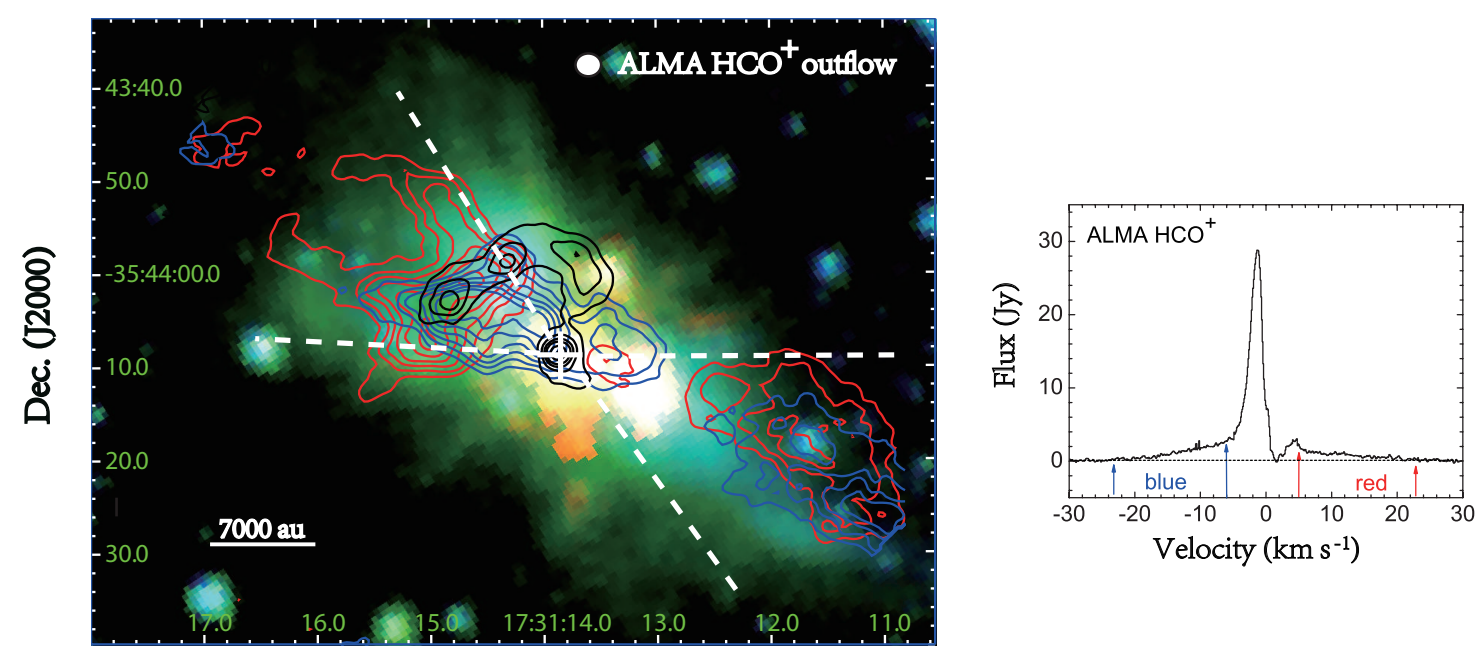

R. A. (J2000)
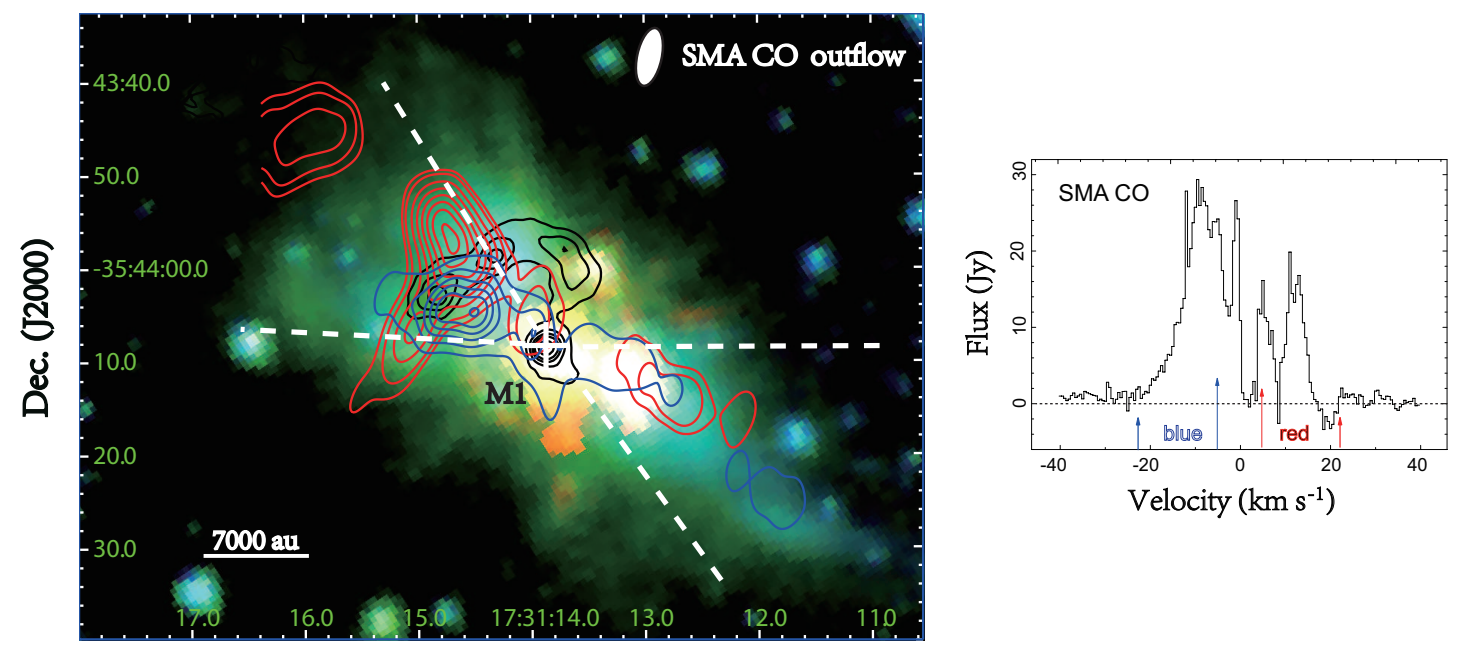

R. A. (J2000)

Figure 2: Molecular outflow images driven by M1 detected with ALMA and SMA. The molecular outflow images (blue contours for blueshifted components and red contours for red-shifted components) are given in left panels, which are obtained from the integrated emission of the corresponding molecules in the blue- and red-shifted velocity ranges given in right panels. The background in the left panels is the three-color GLIMPSE IRAC image same as lower panel of Figure 1, and black contours show the $3 \mathrm{~mm}$ dust emission detected with ALMA, with contours same as Figure 1. Upper panels present the $\operatorname{HCO}^{+}(J=1-0)$ outflow detected with ALMA, with contour levels of $(3,5,7,9,11,13) \times 0.1$ Jy beam $^{-1} \mathrm{~km} \mathrm{~s}^{-1}$. Lower panels show the $\mathrm{CO}(J=2-1)$ outflow detected with SMA, with contour levels of $(1,2,4,6,8,10,12,14) \times 6 \mathrm{Jy}$ beam ${ }^{-1} \mathrm{~km} \mathrm{~s}^{-1}$. The dust component M1 is marked with plus. The beam shapes of the two observations are given by the ellipses. The overall outflow structure of is described by the dashed straight lines. 


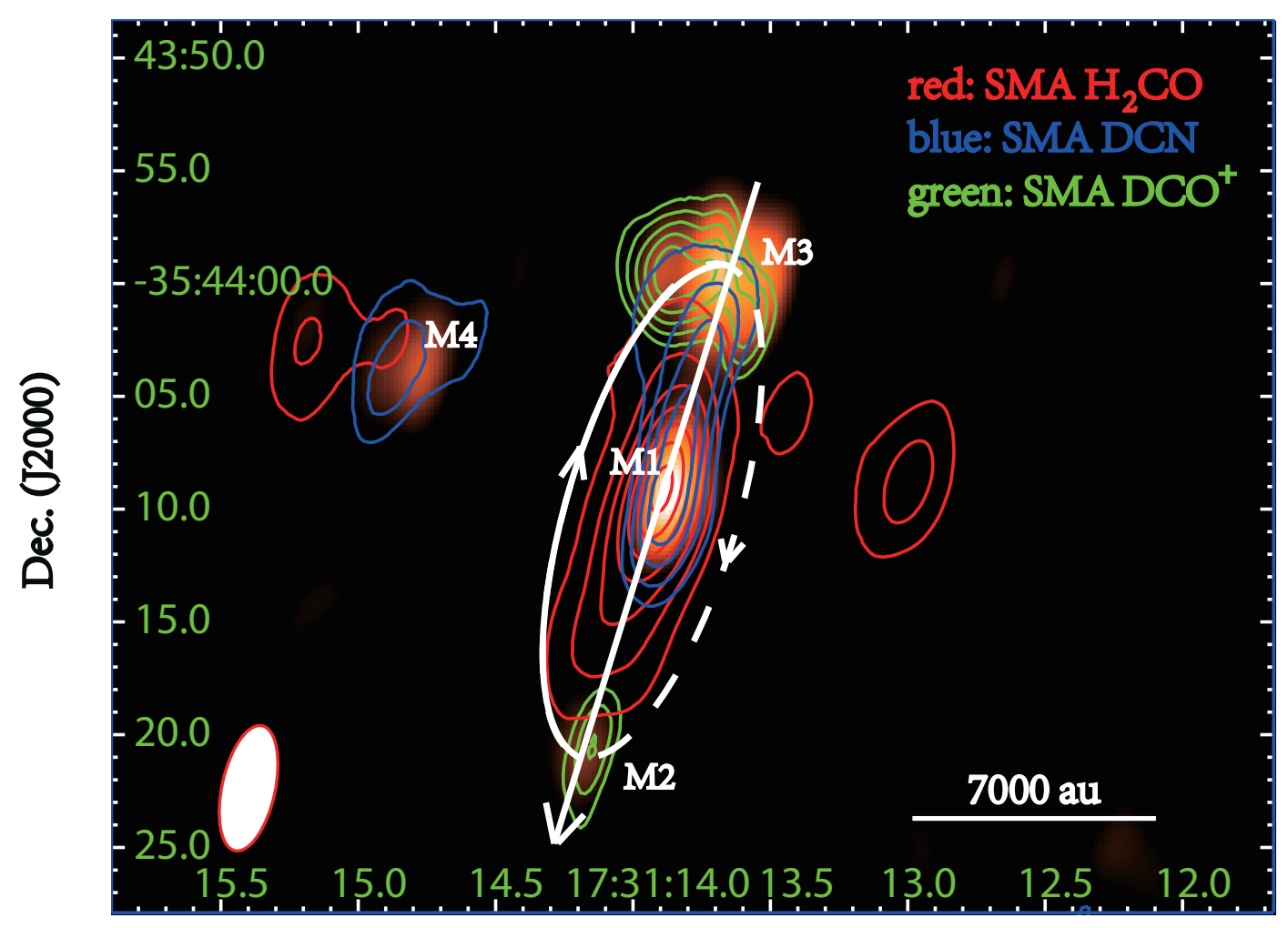

R. A. (J2000)

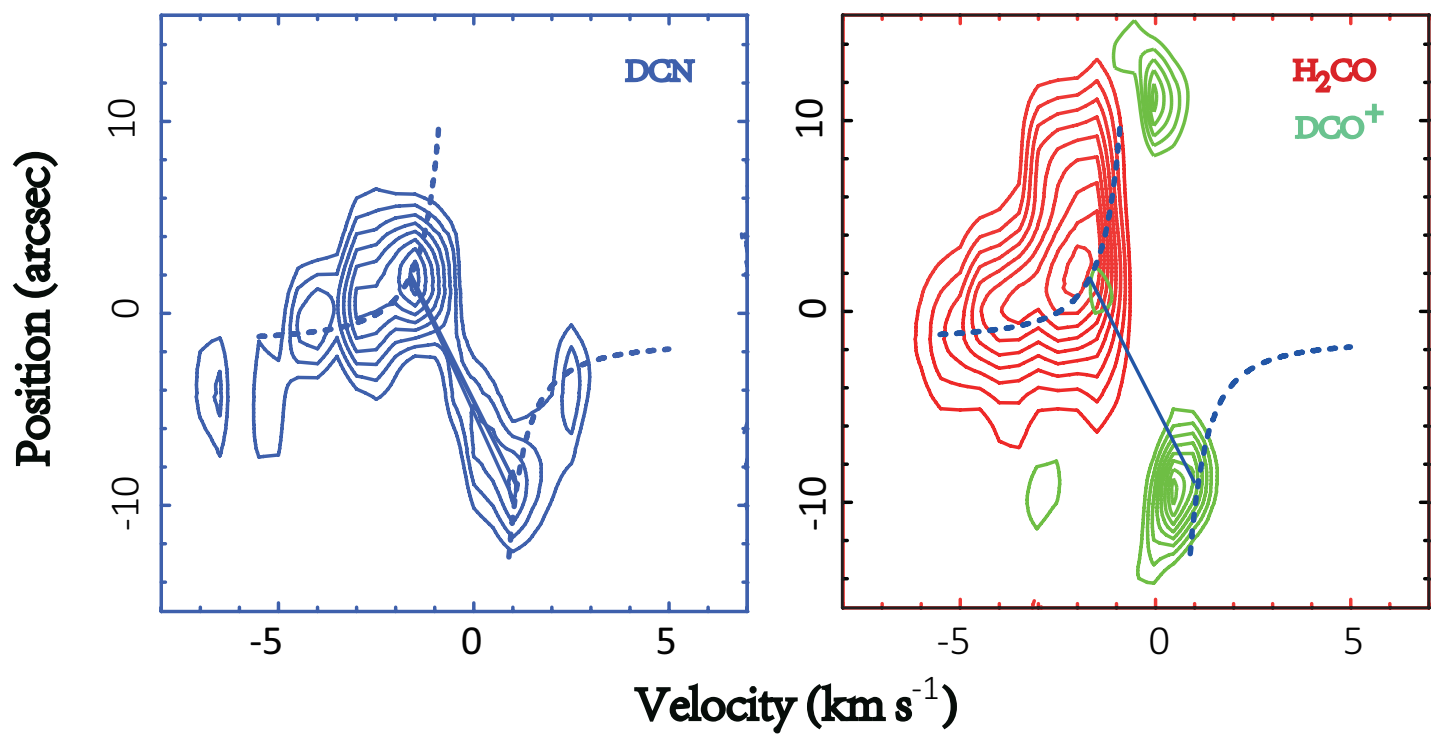

Figure 3: The disk or torus spatial and kinematic structures detected with SMA around M1. Upper panel shows the disk or torus spatial structure traced by integrated emission from molecules $\mathrm{H}_{2} \mathrm{CO} 3(0,3)-2(0,2) 218.222 \mathrm{GHz}$ (red contours), DCN 3-2 217.238 GHz (blue contours) and $\mathrm{DCO}^{+} 4-3288.143 \mathrm{GHz}$ (green contours). The contour levels are: $(3,4,6,8,10) \times 0.5 \mathrm{Jy}_{\text {beam }}^{-1} \mathrm{~km} \mathrm{~s}^{-1}$ for $\mathrm{HCO}^{+} ;(3,4,5,6,7) \times 0.5$ $\mathrm{Jy} \mathrm{beam}^{-1} \mathrm{~km} \mathrm{~s}^{-1}$ for DCN; and $(3,4,5,6,7) \times 0.15 \mathrm{Jy}_{\text {beam }}^{-1} \mathrm{~km} \mathrm{~s}^{-1}$ for DCO ${ }^{+}$. The background is the $1.1 \mathrm{~mm}$ dust emission detected with SMA. The beam shape of the $\mathrm{H}_{2} \mathrm{CO}$ line observation is given by the filled-white ellipse. The white ellipse describes the disk/torus structure suggested by the three molecules and dust emission, with solid half-ellipse meaning towards us. The lower panels show position-velocity (P-V) diagrams for the three molecules along the white line arrow shown in the upper panel. The origin of the P-V diagrams is at the peak of the M1

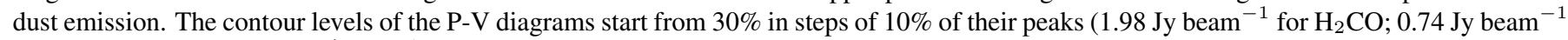
for DCN; and $0.88 \mathrm{Jy}_{\text {beam }}^{-1} \mathrm{DCO}^{+}$). The best Kepler rotation model is shown by blue-dashed curves (see Method). 

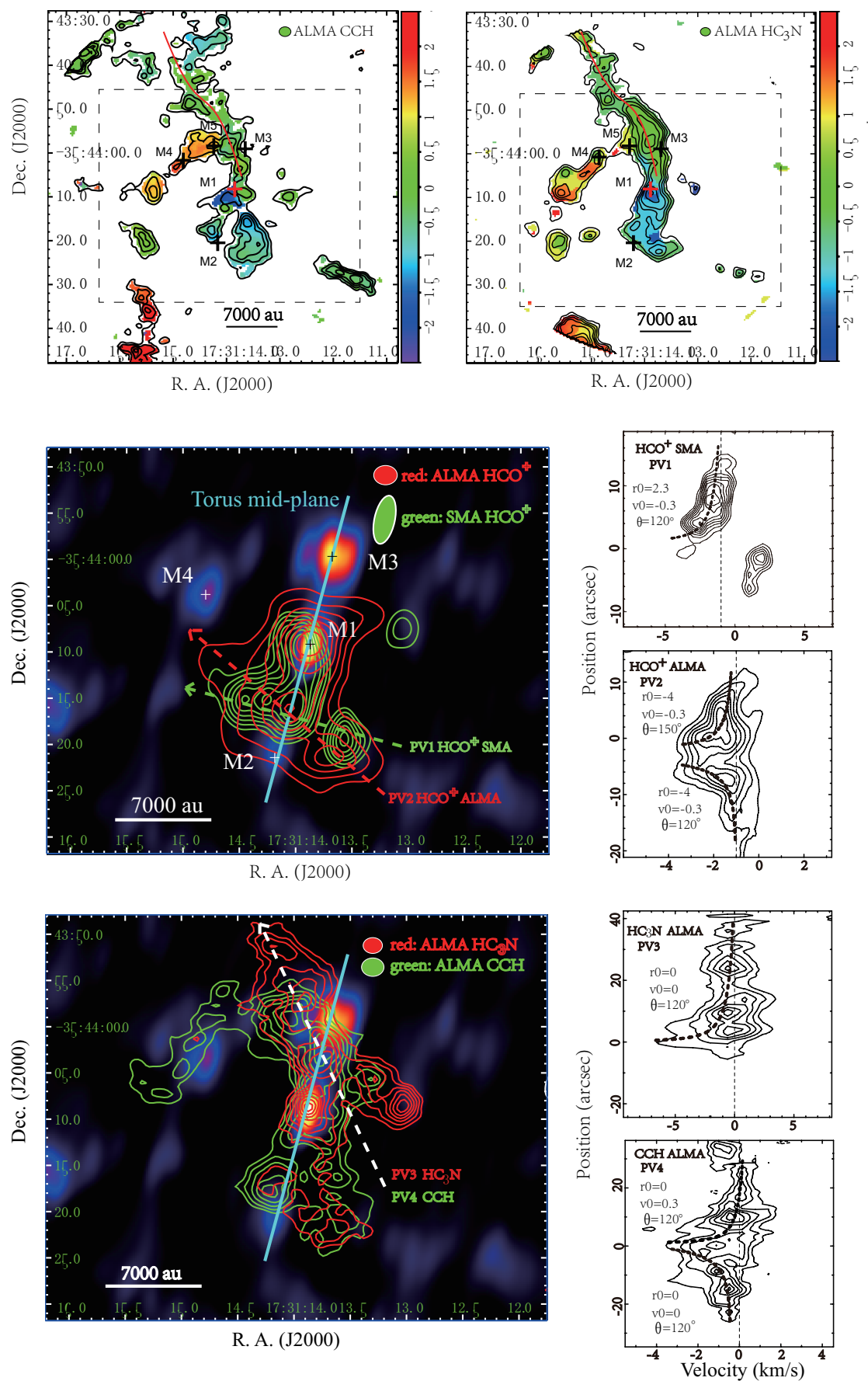

Figure 4: The gravitationally-controlled infalling streamers of chemically fresh molecules detected towards the targeted region. The upper two panels show the images of peak flux density (contours) overlaid the peak velocity (background) for the $\mathrm{CCH} N=1-0, J=3 / 2-1 / 2$, $F=2-1$ transition (left) and the $\mathrm{HC}_{3} \mathrm{~N} J=11-10$ transition (right) detected with ALMA. The contour levels are (3, 4, 5, 6) $\times 0.03$ Jy beam $^{-1}$ for the $\mathrm{CCH}$ and $(3,4,5,6,8,10,12) \times 0.015 \mathrm{Jy}_{\text {beam }}^{-1}$ for the $\mathrm{HC}_{3} \mathrm{~N}$. The red line describes the overall structures of the northeastern infalling streamers of the $\mathrm{CCH}$ and $\mathrm{HC}_{3} \mathrm{~N}$. Middle and lower panels are zoomed-views of two infalling streamers towards the regions defined by the dashed-box shown in the upper panels. Middle left panel presents the southeastern infalling streamer traced by the integrated line emission from the $\mathrm{HCO}^{+}(1-0)$ transition (red contours with levels starting from $3 \mathrm{Jy}$ beam ${ }^{-1} \mathrm{~km} \mathrm{~s}^{-1}$ in steps of $1 \mathrm{Jy} \mathrm{beam}^{-1} \mathrm{~km} \mathrm{~s}^{-1}$ ) and (3-2) transition

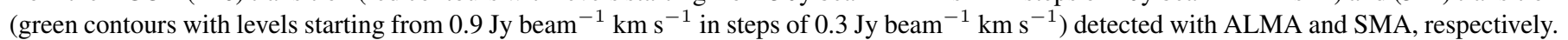
Lower left panel shows the northwestern infalling streamer traced by the integrated line emission from the CCH and $\mathrm{HC}_{3} \mathrm{~N}$ detected with $\mathrm{ALMA}$. The contour levels for both $\mathrm{CCH}$ and $\mathrm{HC}_{3} \mathrm{~N}$ start from $0.24 \mathrm{Jy}$ beam ${ }^{-1} \mathrm{~km} \mathrm{~s}^{-1}$ in steps of $0.08 \mathrm{Jy} \mathrm{beam}^{-1} \mathrm{~km} \mathrm{~s}^{-1}$. The background for the two panels is the $1.1 \mathrm{~mm}$ dust emission detected with SMA. The beam shapes of the two observations are given by the ellipses. The right four panels show the P-V diagrams for the directions labelled in the left two panels. The contour levels of the P-V diagrams start from $30 \%$ in steps of $10 \%$ of their peaks $\left(5.36 \mathrm{Jy}_{\text {beam }}{ }^{-1}\right.$ for the $\mathrm{SMA} \mathrm{HCO}^{+} ; 0.89 \mathrm{Jy}_{\text {beam }}{ }^{-1}$ for the ALMA HCO ${ }^{+} ; 0.12 \mathrm{Jy} \mathrm{beam}^{-1}$ for the ALMA HC$_{3} \mathrm{~N}$; and $0.17 \mathrm{Jy}_{\text {beam }}{ }^{-1}$ for the ALMA CCH). The best free-fall models are shown by dashed curves with the fitted parameters in the corresponding P-V diagrams (see Method). 


\section{METHODS}

Observation and data reduction: ALMA. G352.63-1.07 is one of the target regions in the ATOMS "ALMA Three-millimeter Observations of Massive Star-forming regions" survey (Project ID: 2019.1.00685.S; PI: Tie Liu ${ }^{33}$. The survey sample consists of 146 dense gas clumps selected from IRAS point sources. G352.63-1.07 has source ID 101 in the ATOMS sample and the ALMA observations were made on 2019 December 19. For this source, the observations consist of a single pointing with both the Atacama Compact 7-m Array (ACA; Morita Array) and the 12-m array (C43-2 or C43-3 configurations) in ALMA band 3. The eight spectral windows (SPWs) were tuned to cover 11 commonly observed molecular lines in this frequency range, including those we have focused on in this work $(\mathrm{CCH} 1-0,87.317$ $\mathrm{GHz} ; \mathrm{HCO}^{+} 1-0,89.189 \mathrm{GHz}$ and $\left.\mathrm{HC}_{3} \mathrm{~N} 11-10,100.076 \mathrm{GHz}\right)$. The molecular lines observed with the combined ACA+12-m array is adopted in this work. The SPWs covering the $\mathrm{CCH}$ and $\mathrm{HCO}^{+}$lines have a velocity resolution of $\sim 0.2 \mathrm{~km} \mathrm{~s}^{-1}$, while those covering the $\mathrm{HC}_{3} \mathrm{~N}$ lines have a velocity resolution of $\sim 1.4 \mathrm{~km} \mathrm{~s}^{-1}$. Calibration and imaging were carried out using the CASA software package version 5.6 and for the array configuration we are able to image spatial structures with angular scales of up to $\sim 20$ arcsec in radius. The typical synthesized beam for the reduced combined ACA+12-m array data images is $2.5^{\prime \prime} \times 2.1^{\prime \prime}\left(\mathrm{P} . \mathrm{A} .=88^{\circ}\right)$ for the $\mathrm{CCH}$ and $\mathrm{HCO}^{+}$lines, and is $2.2^{\prime \prime} \times 1.8^{\prime \prime}\left(\mathrm{P} . \mathrm{A} .=88^{\circ}\right)$ for the $\mathrm{HC}_{3} \mathrm{~N}$ lines. The sensitivity of the $\mathrm{ACA}+12-\mathrm{m}$ array images is better than $10 \mathrm{mJy}$ beam $^{-1}$ per channel.

Observation and data reduction: SMA. The SMA observations towards G352.63-1.07 were made on 2017 August 3, with the array in its compact configuration. The SWARM correlator was used in dual receiver mode with an $8-\mathrm{GHz}$ bandwidth per sideband. The $230 \mathrm{GHz}$ and $240 \mathrm{GHz}$ receivers were used to cover the main targeted lines in this work, which included ${ }^{12} \mathrm{CO}(2-1 ; 230.538 \mathrm{GHz}), \mathrm{HCO}^{+}(3-2 ; 267.557$ $\mathrm{GHz}), \mathrm{DCN}(3-2 ; 217.238 \mathrm{GHz}), \mathrm{DCO}^{+}(4-3 ; 288.143 \mathrm{GHz})$, and a series of $\mathrm{CH}_{3} \mathrm{CN}(12-11 ; \sim 220 \mathrm{GHz})$, and $\mathrm{H}_{2} \mathrm{CO}(3-2 ; \sim 218 \mathrm{GHz})$ lines. The spectral channel width was about $0.5 \mathrm{MHz}$, corresponding to a velocity resolution of $\sim 0.7 \mathrm{~km} \mathrm{~s}^{-1}$ at $230 \mathrm{GHz}$, and $\sim 0.5 \mathrm{~km} \mathrm{~s}^{-1}$ at $280 \mathrm{GHz}$. The time dependent antenna gains were calibrated using regular observations of the quasar 1700-261. The bandpass response was calibrated by observing the quasar 3C454.3 and the absolute flux density was determined from observations of Neptune and Titan.

The visibility data were calibrated using the IDL superset MIR. The imaging and analysis were undertaken in MIRIAD. The synthesized beam size, i.e., the angular resolution of the image data are approximately $5.8^{\prime \prime} \times 2.4^{\prime \prime}$ at $230 \mathrm{GHz}$ and $4.7^{\prime \prime} \times 1.9^{\prime \prime}$ at $285 \mathrm{GHz}$, with a position angle of $-12.3^{\circ}$ northwest. The typical rms noise is $\sim 50$ mJy beam ${ }^{-1}$ in a spectral channel for the molecular line data. We used the line-free channels in the frequency range $281-289 \mathrm{GHz}$ to produce the $1.1 \mathrm{~mm}$ dust emission image. The rms noise for the dust emission image is $\sim 4$ mJy beam $^{-1}$.

Gas temperature in the hot molecular core in M1. Hot molecular core tracers (such as $\mathrm{CH}_{3} \mathrm{CN}$ and $\mathrm{CH}_{3} \mathrm{OH}$ ) were only detected towards a compact region (not resolved by either the ALMA nor SMA observations) towards the dust component M1. We have assumed that the molecular gas is in local thermodynamic equilibrium (LTE) to estimate physical parameters. The column density can be calculated using a general formula ${ }^{34,35}$

$N=\frac{3 k W}{8 \pi^{3} \nu S \mu^{2}}\left(\frac{T_{e x}}{T_{e x}-T_{b g}}\right)\left(\frac{\tau}{1-\exp (-\tau)}\right) Q\left(T_{e x}\right) \exp \left(E_{u} / k T_{e x}\right)$,

where $k$ is the Boltzmann constant in erg $\mathrm{K}^{-1}, W$ is the observed line integrated intensity of the species in units of $\mathrm{K} \mathrm{km} \mathrm{s}^{-1}, \nu$ is the frequency of the transition in $\mathrm{Hz}$, and $S \mu^{2}$ is the product of the total torsion-rotational line strength and the square of the electric dipole mo- ment, $E_{u} / k$ is the upper level energy in $\mathrm{K}$ and $\mathrm{Q}\left(\mathrm{T}_{e x}\right)$ is the partition function at temperature $T_{e x}$.

When the molecular emission is optically thin and in LTE, we can determine the rotation temperature $\left(\mathrm{T}_{r o t}\right)$ from rotation diagram analysis. The standard formula for such analysis can be derived from Equation (1) as follows:

$$
\ln \left(\frac{3 \kappa W}{8 \pi^{3} \nu S \mu^{2}}\right)=\ln \frac{N\left(T_{\text {rot }}-T_{b g}\right)}{Q\left(T_{\text {rot }}\right)}-\frac{E_{u} / \kappa}{T_{\text {rot }}} .
$$

A straight line fitted to a plot of $\ln \left(3 \kappa W / 8 \pi^{3} \nu S \mu^{2}\right)$ versus $E_{u} / \kappa$ will have a slope of $1 / T_{\text {rot }}$. We used eight transitions of $\mathrm{CH}_{3} \mathrm{CN}$ (from $12_{0}-11_{0}$ to $12_{7}-11_{7}$ with frequencies in the range $220.5-220.8 \mathrm{GHz}$ ) detected in the SMA observations to estimate the rotation temperature of the hot gas in M1 (see Extended Figure 1). The derived gas rotation temperature is $165 \pm 45 \mathrm{~K}$, confirming that M1 corresponds to a hot molecular core.

Kepler rotation model for the M1-M2-M3 disk/torus system. In the case of system undergoing Keplerian rotation, the speed of rotation along the line-of-sight for a given enclosed mass $(M)$ and radius $(R)$ along the disk/torus satisfies the following relation:

$$
\frac{v_{L O S}-v_{\star}}{\sin \theta}=\sqrt{\frac{G M}{R-R_{0}}},
$$

where $G$ is the gravitational constant, $v_{\star}$ is the systemic velocity of the disk/torus system, $\theta$ is the inclination angle of the disk/torus rotational axis, and $R_{0}$ is the offset from the zero position in the $\mathrm{P}-\mathrm{V}$ diagram (i.e. the $\mathrm{M} 1$ position). By adjusting the parameters $\mathrm{M}, v_{\star}$ and $R_{0}$ to fit the $\mathrm{P}$ $\mathrm{V}$ diagrams of the disk tracers $\mathrm{H}_{2} \mathrm{CO}$ and $\mathrm{DCN}$, we find $\mathrm{M}=9.0 / \sin ^{2} \theta$ $\mathrm{M}_{\odot}, v_{\star}=-0.3 \mathrm{~km} \mathrm{~s}^{-1}$ and $R_{0}=-1000$ au (or $-1.5^{\prime \prime}$ ) provide the best fits to the data. Furthermore, if we assume an inclination angle of $\theta=\sim 60^{\circ}$ (based on the distorted structures traced by $\mathrm{DCO}^{+}$and dust emission at M3), we then estimate an enclosed mass of $\sim 12 \mathrm{M}_{\odot}$ in this rotational system. It should be noted that in our fit, $R_{0}=-1000 \mathrm{au}$, so the gravitational center of the rotational system is not at the M1 peak, but at position of 1000 au northward away from the M1 peak along the $\mathrm{P}-\mathrm{V}$ slice. This may be caused by inhomogeneous material distribution in the disk/torus system.

Mass estimated from the $\mathbf{1 . 1} \mathbf{~ m m}$ dust emission. We estimated the rotational system mass using the $1.1 \mathrm{~mm}$ dust emission under the assumption that it is optically thin. Under these conditions, the mass can be found using the relation

$$
M_{\text {gas }}=\frac{S_{\text {int }} D^{2}}{\kappa_{d} B_{\nu}\left(T_{\text {dust }}\right) R_{d}},
$$

where $S_{\text {int }}$ is the integrated flux density of the $1.1 \mathrm{~mm}$ dust emission, $D$ is the distance to the source, $\kappa_{d}$ is the mass absorption coefficient per unit mass of dust, $B_{\nu}\left(T_{\text {dust }}\right)$ is the Planck function at temperature $T_{d u s t}$, and $R_{d}$ is the dust-to-gas mass ratio. Here we have used $\kappa_{d}=1.14 \mathrm{~cm}^{2} \mathrm{~g}^{-1}$ for $1.1 \mathrm{~mm}$ (ref. 36) and a dust-to-gas ratio $\left(R_{d}\right)$ of $1: 100$ in our calculations.

For M1 $\left(S_{\text {int }}=0.1 \mathrm{Jy}\right)$, which is a hot molecular core with a gas temperature of $\sim 165 \mathrm{~K}$ (see above), we have assumed the dust temperature $\left(\mathrm{T}_{d u s t}\right)$ is equal to the gas temperature under LTE. Based on this, we estimate that the mass of M1 is only $0.05 \mathrm{M}_{\odot}$. Even considering a dust temperature of $20 \mathrm{~K}$, the mass of $\mathrm{M} 1$ is $\sim 0.6 \mathrm{M}_{\odot}$. These are inconsistent with a high-mass protostar and more likely to trace the mass of the internal hot disk around protostar embedded in M1.

For M3 $\left(S_{\text {int }}=0.15 \mathrm{Jy}\right)$, located at the disk/torus edge, a cold environment we have assumed a dust temperature of $10 \mathrm{~K}$, which is equal to the cold gas temperature derived from the $\mathrm{CCH}$ observations (see below). Under these assumptions we estimate the mass in the region is about $2.5 \mathrm{M}_{\odot}$. Considering that the gravitation center of the disk/torus 
rotational system has an offset $R_{0}=-1.5^{\prime \prime}$ from M1 towards M3, we can estimate a total mass of the rotational system to be $\sim 15 \mathrm{M}_{\odot}$ according the motion of the center of mass. This is consistent with that derived from the Keplerian rotation model (see above). Excluding the mass contributions from the outer torus traced by M2 and M3, the mass of the protostar embedded in M1 is likely in the range $10-12 \mathrm{M}_{\odot}$.

Gas temperature distribution along the disk/torus. The gas kinetic temperatures along the disk/torus are estimated from the line ratios of para- $\mathrm{H}_{2} \mathrm{CO} 3_{03}-2_{02} / 3_{22}-2_{21}$ (ref. 37) by performing the nonlocal thermodynamic equilibrium (non-LTE) radiation transfer code RADEX $^{38}$ coupled with collision rates from the Leiden Atomic and Molecular Database calculated by the ref. 39. In the calculations, the number density of molecular hydrogen is $10^{5} \mathrm{~cm}^{-3}$, the column density of para- $\mathrm{H}_{2} \mathrm{CO}$ is $5 \times 10^{13} \mathrm{~cm}^{-2}$, the line width is $2 \mathrm{~km} \mathrm{~s}^{-1}$ and the background temperature is $2.73 \mathrm{~K}$. The line ratios lower than 1.67 corresponding to the temperatures higher than $360 \mathrm{~K}$ are totally ignored because the line ratios of para- $\mathrm{H}_{2} \mathrm{CO} 3_{03}-2_{02} / 3_{22}-2_{21}$ are not sensitive for gas kinetic temperature measurements. Extended figure 2 shows the derived gas kinetic temperature distributions and gradient along the disk/torus plane. Notably, we can not determine the temperatures towards northwester region of the $\mathrm{H}_{2} \mathrm{CO}$ emission, because the above method is invalid due to the lower ratios between the two lines.

Gravitationally-controlled infalling streamers. In the case of gravitationally-controlled infall, it is possible to estimate the speed of free-fall for an enclosed mass $(M)$ and radius $(R)$ using the relation:

$$
\frac{v_{L O S}-v_{0}}{\cos \theta}=\sqrt{\frac{2 G M}{R-R_{0}}},
$$

where $\theta$ is the inclination angle of the streamer along the line-of-sight, $v_{0}$ is the velocity at the starting position of the streamer, and $R_{0}$ is the projection height of the landing point of the streamer onto the disk/torus. We have assumed $M \sim 12 M_{\odot}$, the mass derived from the the rotational disk/torus system and adjusted the parameters $\theta, v_{0}$ and $R_{0}$ to fit the P-V distributions of the infalling streamers shown in Figure 4. These P-V diagrams can be well fit using this method, suggesting that the streamers are moving towards the disk/torus under gravitational attraction. The values estimated for $\theta, v_{0}$ and $R_{0}$ are different for each streamer, indicating that the infalling directions of these streamers and the landing point on the disk/torus are different.

The free-fall timescale of the infalling streamers can be derived as

$$
t_{f f}=\sqrt{\frac{R^{3}}{G M}}
$$

For these streamers, the typical observed length $R$ in the sky-plane is equal to $\sim 1.0 \times 10^{4}$ au, and for $M=12 \mathrm{M}_{\odot}$, the free-fall timescale is $\sim 4.6 \times 10^{4}$ years.

Cold gas properties of the streamers. Under LTE conditions, we assume an excitation temperature $\left(\mathrm{T}_{e x}\right)$ equal to the gas kinematic temperature. We can derive the excitation temperatures from the $\mathrm{CCH}$ observations (one of the chemically fresh molecules in the streamers) using the ratio between its two hyperfine lines $(N=1-0, J=$ $3 / 2-1 / 2, F=2-1 ; N=1-0, J=3 / 2-1 / 2, F=1-0)$. Firstly, the optical depth of the main line $(F=2-1)$ can be estimated using the following relation:

$$
\frac{T_{B, s}}{T_{B, m}}=\frac{1-\exp \left(-0.5 \tau_{m}\right)}{1-\exp \left(-\tau_{m}\right)}
$$

where $T_{B, m}$ and $T_{B, s}$ are the observed brightness temperature in $\mathrm{K}$ for the main and satellite lines, respectively. We have used that the expected opacity ratio $(r)$ between the main and satellite lines is $\tau_{m} / \tau_{s}=2$, under optically thin conditions. We can further calculate the excitation temperature using the relation:

$$
T_{B}=T_{0}\left(\frac{1}{e^{T_{0} / T_{e x}}-1}-\frac{1}{e^{T_{0} / T_{b g}}-1}\right)\left(1-e^{-\tau}\right),
$$

where $T_{b g}(=2.73 \mathrm{~K})$ is the background brightness temperature and $T_{0}=h \nu / k$.

We obtain the ratio between the peak intensities of the $\mathrm{CCH}$ maps of the two hyperfine transitions (from ALMA observations) to calculate the optical depth and excitation temperature in each pixel. The derived excitation temperature distributions are illustrated in Extended Figure 2. The excitation temperatures are the range 8-20 K, with a typical value of $\sim 10 \mathrm{~K}$. The streamers can almost be traced by the $\mathrm{CCH}$ (in particular for the northern one), indicating that the streamers are chemically fresh and in a cold environment.

Infall material rates of the streamers. Based on the optical depth $(\tau)$ and excitation temperature $\left(T_{e x}\right)$, we can determine the column density in the streamer regions. Using Eq. (1). we can derive the column density in each pixel of the $\mathrm{CCH}$ streamer. We summed the $\mathrm{CCH}$ gas mass in the northwestern streamer region and estimate the total gas mass to be $\sim 2.5 \mathrm{M}_{\odot}$ (assuming a typical $\mathrm{CCH}$ abundance of $\left[\mathrm{CCH} / \mathrm{H}_{2}\right]=8 \times 10^{-9}$ (ref. 23)) and a mean mass per particle of $\mu=2.33 m_{H}$ (ref. 40 ).

The $\mathrm{CCH}$ emission associated with the southeastern streamer is much weaker. Using Eq. (1), we have derived the gas mass along this streamer using the ALMA $\mathrm{HCO}^{+}(3-2)$ observations, assuming the emission is optically thin and at a gas temperature of $10 \mathrm{~K}$. This gives a total gas mass of $\sim 1.5 \mathrm{M}_{\odot}$ in the south-eastern streamer region, assuming a typical $\mathrm{HCO}^{+}$abundance of $\left[\mathrm{HCO}^{+} / \mathrm{H}_{2}\right]=1.2 \times 10^{-9}$ (ref. 30)

Using the calculated free-fall timescale of $4.6 \times 10^{4}$ years, we can estimate the infall material rates along these streamers to be about $5.5 \times$ $10^{-5} \mathrm{M}_{\odot}$ year $^{-1}$ for the northwestern stream and $3.1 \times 10^{-5} \mathrm{M}_{\odot}$ year $^{-1}$ for the southeastern stream. This implies a total of infall rate of $8.6 \times 10^{-5} \mathrm{M}_{\odot}$ year $^{-1}$ from the two streamers.

Assuming the that accreting timescale is equal to the free-fall timescale of the infalling streamer $\left(4.6 \times 10^{4}\right.$ years; see above), and that the mass of the protostar in $\mathrm{M} 1$ is about $\sim 10 \mathrm{M}_{\odot}$, this suggests that the overall accretion rate is $\sim 2.1 \times 10^{-4} \mathrm{M}_{\odot}$ year $^{-1}$. Therefore, it also suggests that the detected infalling streamers can contribute up to $40 \%$ of the material in the accretion process.

Mass-loss rate from the outflow driven by M1. We estimate the mass-loss rate from the outflow driven by M1 using the $\mathrm{CO}$ outflow. Assuming that the $\mathrm{CO}$ emission in the outflow lobes is optically thin at a gas temperature of $20 \mathrm{~K}$ (which is a typical temperature in a cold $\mathrm{CO}$ gas region, and a bit higher than the $\mathrm{CO}$ evaporation temperature of $15 \mathrm{~K}$; ref. 41), we can use Eq. (1) to derive the column density for each pixel. A total of mass of $\sim 0.05 \mathrm{M}_{\odot}$ is obtained summing over the $\mathrm{CO}$ emission in the northeastern outflow lobe region under the assumption of a $\mathrm{CO}$ abundance of $\left[\mathrm{CO} / \mathrm{H}_{2}\right]=1.0 \times 10^{-4}$. If we consider the masses obtained for the blue- and red-shifted components are nearly same and a dynamic age of $\sim 3600$ years for the outflow (see main text), it suggests a mass-loss rate of $\sim 1.4 \times 10^{-5} \mathrm{M}_{\odot}$ year $^{-1}$ from the outflow driven by M1. Notably, considering that part of CO emission in the outflow regions may be optically thick and mass of the energetic part of the outflow traced by the infrared emission, the above estimation of mass-loss rate can be treated as a lower limit.

Data availability The data from both ALMA and SMA that support the plots within this paper and other findings of this study are available from the corresponding author upon reasonable request. 
33. Liu, T. et al. ATOMS: ALMA three-millimeter observations of massive starforming regions - I. Survey description and a first look at G9.62+0.19. Mon. Not. R. Astron. Soc. 496, 2790-2820 (2020).

34. Blake, G. A., Sutton, E. C., Masson, C. R., \& Phillips, T. G. Molecular abundances in OMC-1: The chemical composition of interstellar molecular clouds and the influence of massive star formation. Astrophys. J. 315, 621-645 (1987).

35. Chen, $X$. et al. Newly identified extended green objects (EGOs) from the Spitzer GLIMPSE II Survey. I. Catalog Astrophys. J. Supp. 206, 9 (2013).

36. Ossenkopf, V. \& Henning, Th. Dust opacities for protostellar cores. Astron. Astrophys. 291, 943-959 (1994).

37. Mangum, J. G., Wootten, A., Plambeck, R. L. The physical structure of Orion-KL on $2500 \mathrm{AU}$ scales using the K-doublet transitions of formaldehyde. Astrophys. J. 409, 282-298 (1993).

38. van der Tak, F. F. S., Black, J. H., Schöier, F. L., Jansen, D. J., van Dishoeck, E. F. A computer program for fast non-LTE analysis of interstellar line spectra. With diagnostic plots to interpret observed line intensity ratios. Astron. Astrophys. 468, 627-635 (2007).

39. Wiesenfeld, L., Faure, A. Rotational quenching of $\mathrm{H}_{2} \mathrm{CO}$ by molecular hydrogen: cross-sections, rates and pressure broadening. Mon. Not. $R$ Astron. Soc. 432, 2573-2578 (2013).

40. Myers, P. C. \& Benson, P. J. Dense cores in dark clouds. II. $\mathrm{NH}_{3}$ observations and star formation. Astrophys. J. 266, 309-320 (1983).

41. Bergin, E. A. \& Tafalla, M. Cold dark clouds: the initial conditions for star formation. Annu. Rev. Astron. Astrophys. 45, 339C-396 (2007). 


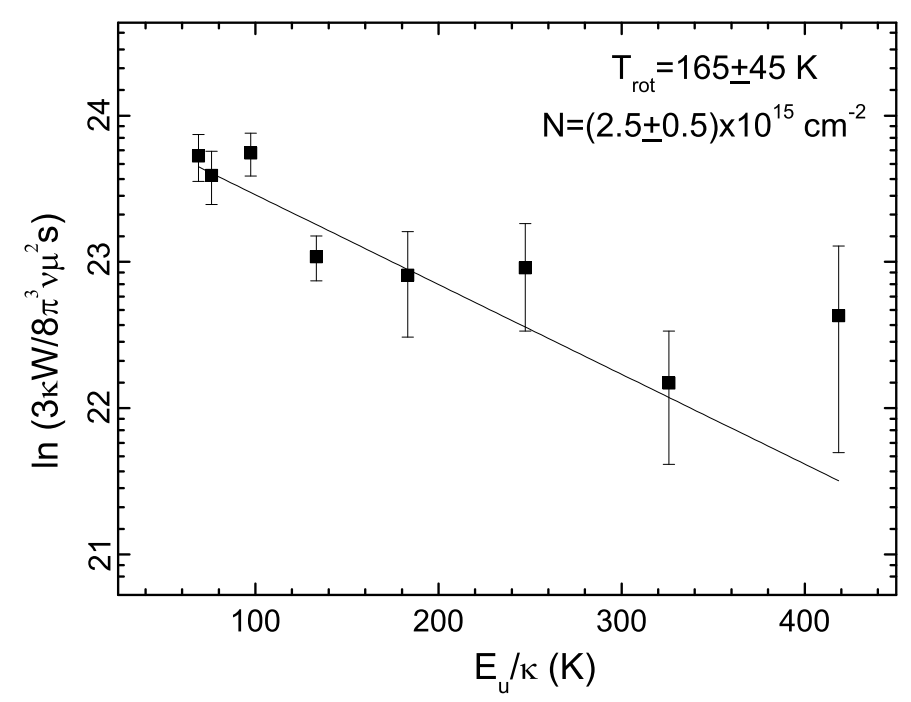

Extended Data Fig 1. - The $\mathrm{CH}_{3} \mathrm{CN}$ rotation diagram of the M1 core. The solid line is the best fit to the rotation diagram, and the fit results are shown in the top right.

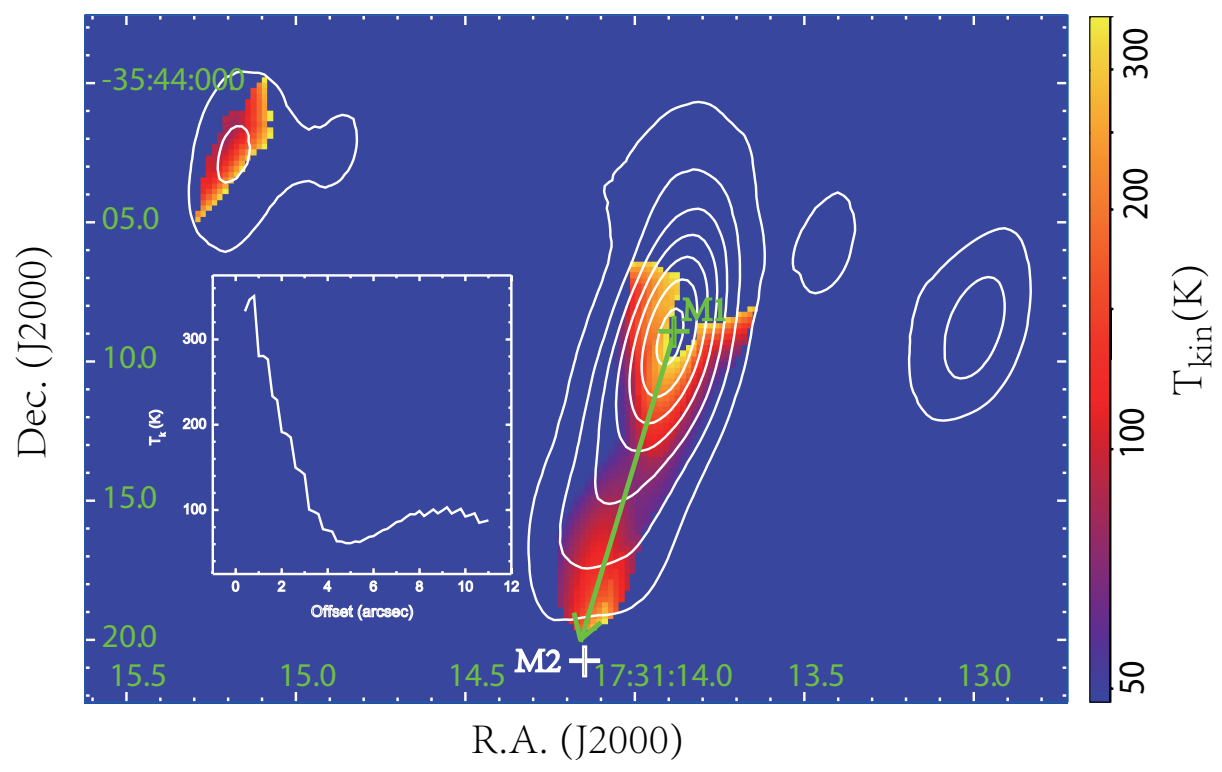

Extended Data Fig 2. - The distributions of the kinematics temperatures of the $\mathrm{H}_{2} \mathbf{C O}$ along the disk/torus direction. Background is the derived kinematics temperature of the $\mathrm{H}_{2} \mathrm{CO}$. White contours show integrated flux densities of the $3(0,3)-2(0,2) 218.222 \mathrm{GHz} \mathrm{H}_{2} \mathrm{CO}$ transition, with contour levels same as that given in the upper panel of Figure 3. The insert panel show the kinematics temperature gradient along the disk/torus direction. 


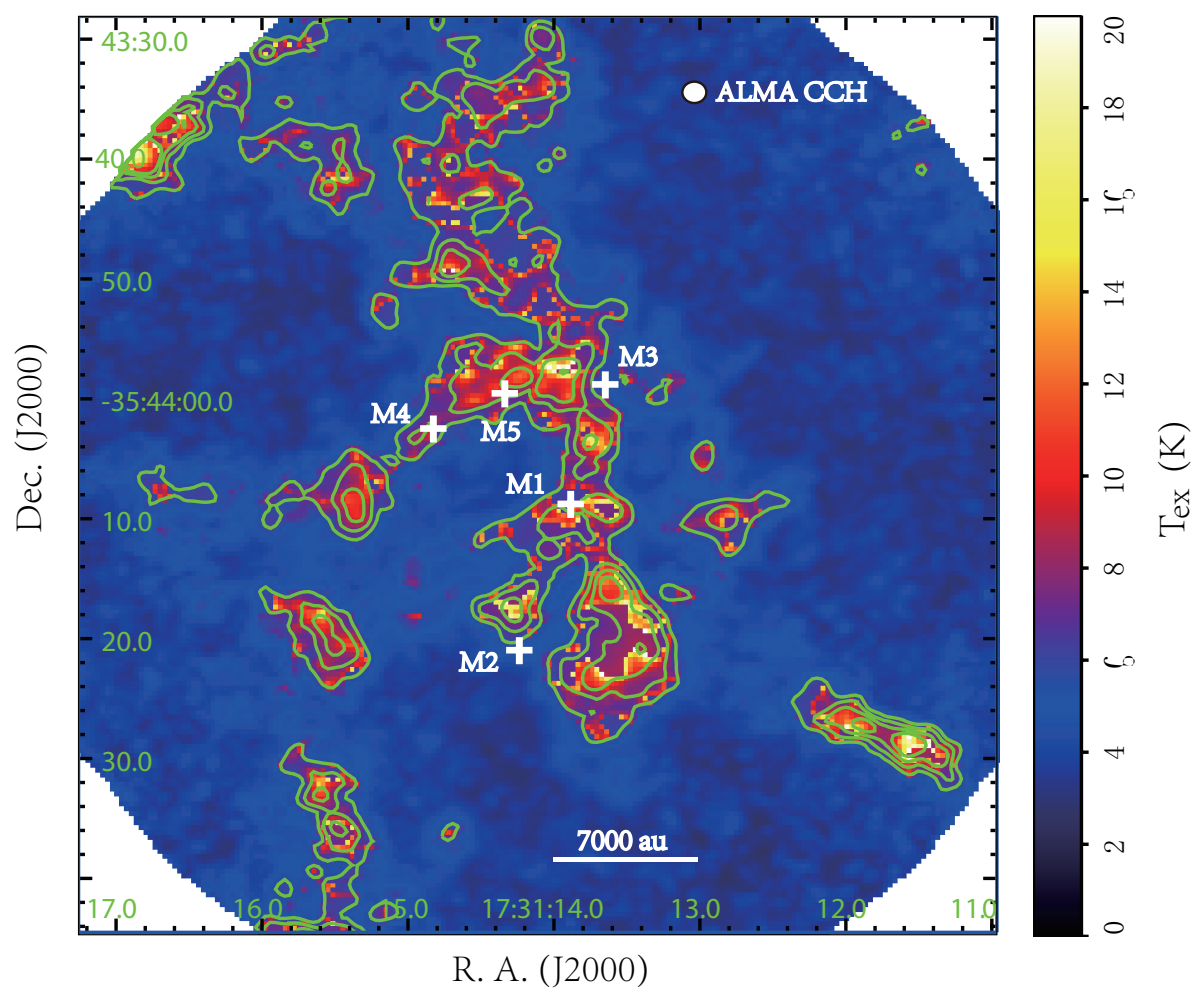

Extended Data Fig 3. - The excitation temperatures of the $\mathrm{CCH}$ derived from its two hyperfine lines. Background is the derived excitation temperature of the $\mathrm{CCH}$. Green contours show peak flux densities of the $\mathrm{CCH} N=1-0, J=3 / 2-1 / 2, F=2-1$ transition, with contour levels same as that given in the upper panel of Figure 4. The beam shape of the ALMA observation is given by the ellipse. 


\section{Figures}

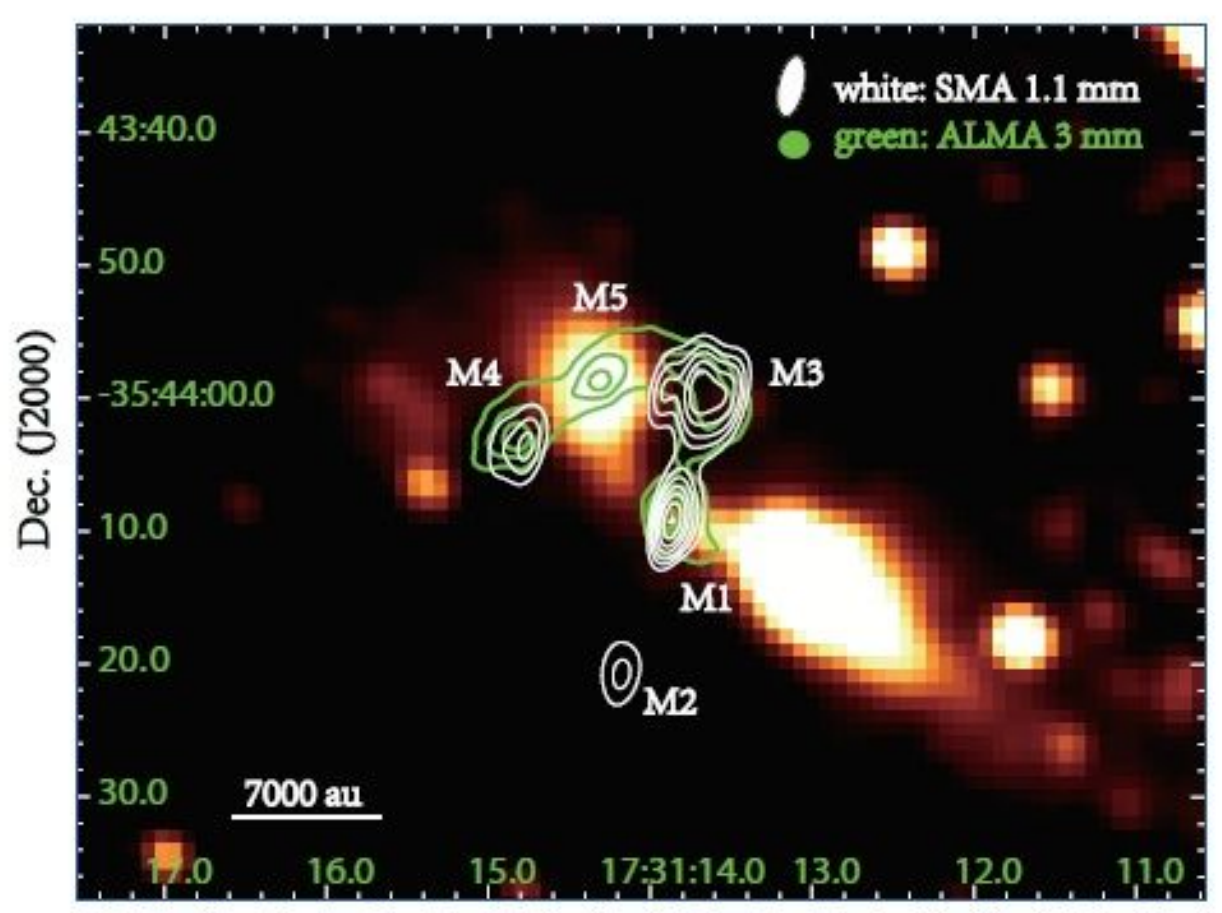

R. A. (J2000)

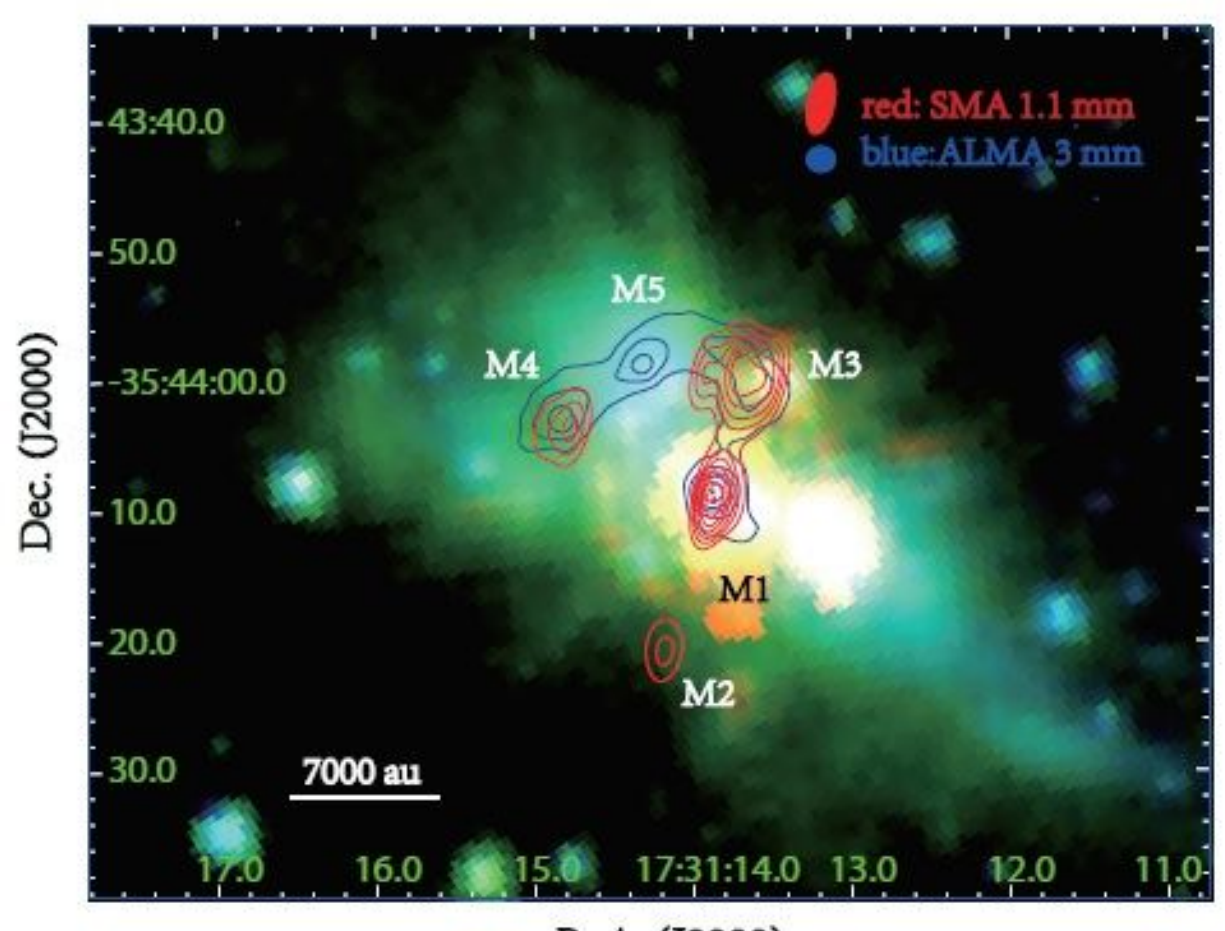

R. A. (J2000)

Figure 1

Millimeter dust emission maps imaged with ALMA and SMA towards G352.63-1.07. Green and blue contours (in upper and lower panels, respectively) show the $3 \mathrm{~mm}$ dust emission detected with ALMA, with contour levels of $(1,2,3,4,6,8)-1$ mJy beam-1. White and red contours (in upper and lower panels, 
respectively) show the $1.1 \mathrm{~mm}$ dust emission detected with SMA, with contour levels of $(3,5,7,10,13,16$, 19)u4 mJy beam-1. The corresponding dust emission components are marked with M1-M5. The beam shapes of the two observations are given by the ellipses. The background are the near-infrared 2MASS Ks 2 um for upper panel, and the three-color GLIMPSE IRAC image composite with 8.0 um in red, 4.5 um in green, and 3.6 um in blue for lower panel.
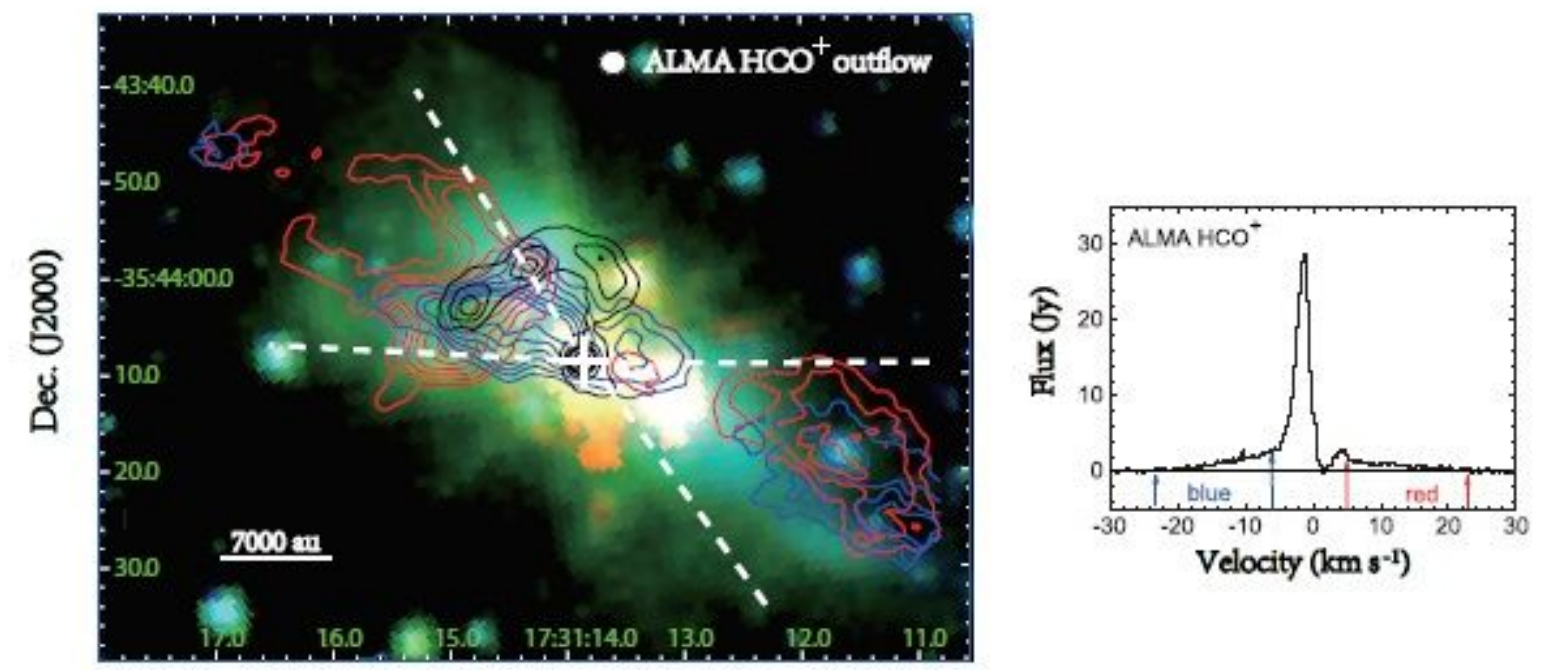

\section{R. A. (J2000)}
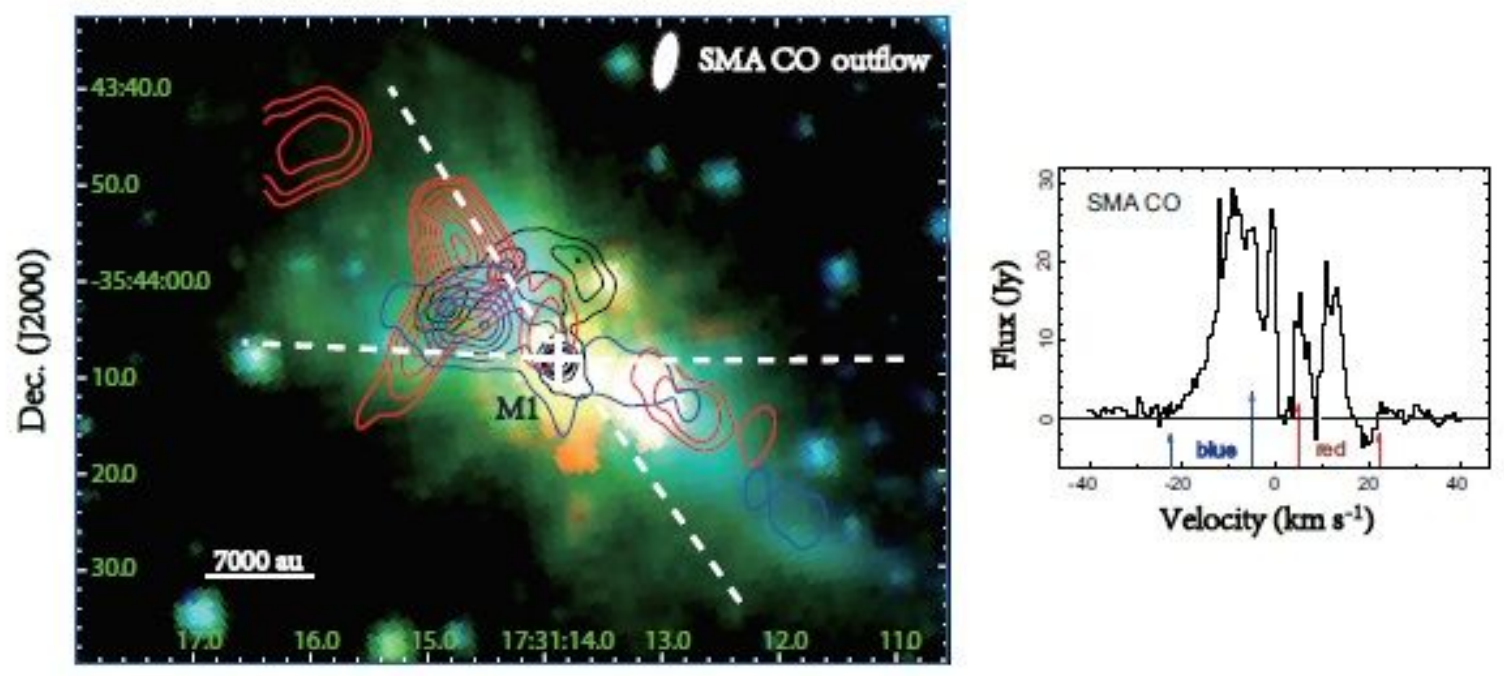

R. A. (J2000)

\section{Figure 2}

Molecular outflow images driven by M1 detected with ALMA and SMA. The molecular outflow images (blue contours for blueshifted components and red contours for red-shifted components) are given in left panels, which are obtained from the integrated emission of the corresponding molecules in the blue- and red-shifted velocity ranges given in right panels. The background in the left panels is the three-color GLIMPSE IRAC image same as lower panel of Figure 1, and black contours show the $3 \mathrm{~mm}$ dust emission detected with ALMA, with contours same as Figure 1. Upper panels present the $\mathrm{HCO}+(\mathrm{J}=1-0)$ outflow detected with ALMA, with contour levels of $(3,5,7,9,11,13) \times 0: 1$ Jy beam-1 km s-1. Lower panels show 
the CO $(\mathrm{J}=2$ - 1$)$ outflow detected with SMA, with contour levels of $(1,2,4,6,8,10,12,14) \times 6 \mathrm{Jy}$ beam-1 $\mathrm{km} \mathrm{s}-1$. The dust component M1 is marked with plus. The beam shapes of the two observations are given by the ellipses. The overall outflow structure of is described by the dashed straight lines.
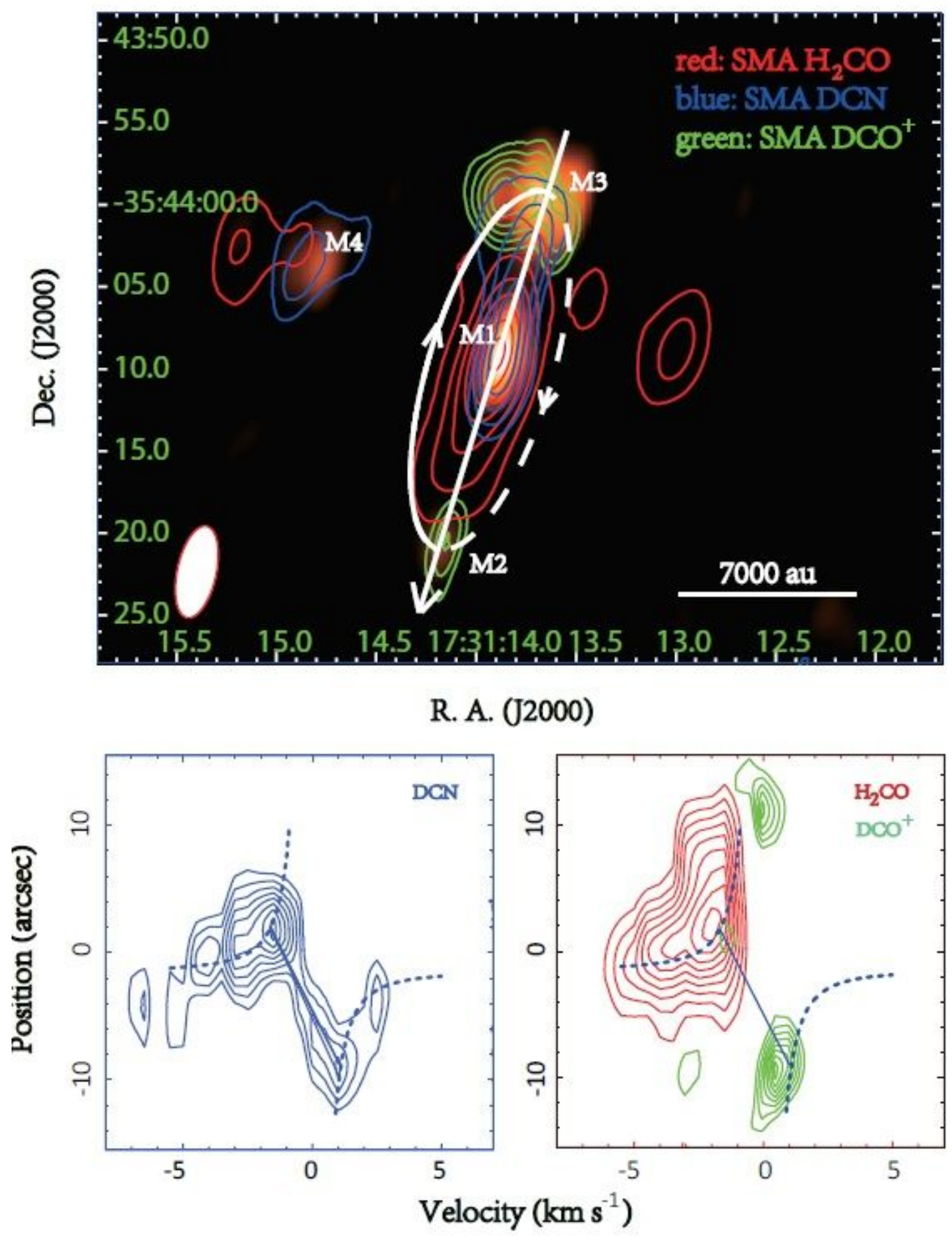

Figure 3

The disk or torus spatial and kinematic structures detected with SMA around M1. Upper panel shows the disk or torus spatial structure traced by integrated emission from molecules H2CO 3(0,3)-2(0,2) 218.222 $\mathrm{GHz}$ (red contours), DCN 3-2 217.238 GHz (blue contours) and DCO+4-3 288.143 GHz (green contours). 
The contour levels are: $(3,4,6,8,10) \times 0: 5 \mathrm{Jy}$ beam $-1 \mathrm{~km} \mathrm{~s}-1$ for HCO+; $(3,4,5,6,7) \times 0: 5 \mathrm{Jy}$ beam-1 km s-1 for DCN; and $(3,4,5,6,7) \times 0: 15$ Jy beam-1 km s-1 for DCO+. The background is the $1.1 \mathrm{~mm}$ dust emission detected with SMA. The beam shape of the $\mathrm{H} 2 \mathrm{CO}$ line observation is given by the filled-white ellipse. The white ellipse describes the disk/torus structure suggested by the three molecules and dust emission, with solid half-ellipse meaning towards us. The lower panels show position-velocity (P-V) diagrams for the three molecules along the white line arrow shown in the upper panel. The origin of the P$\mathrm{V}$ diagrams is at the peak of the M1 dust emission. The contour levels of the P-V diagrams start from $30 \%$ in steps of $10 \%$ of their peaks ( $1.98 \mathrm{Jy}$ beam- 1 for $\mathrm{H} 2 \mathrm{CO}$; $0.74 \mathrm{Jy}$ beam -1 for DCN; and $0.88 \mathrm{Jy}$ beam-1 DCO+). The best Kepler rotation model is shown by blue-dashed curves (see Method).

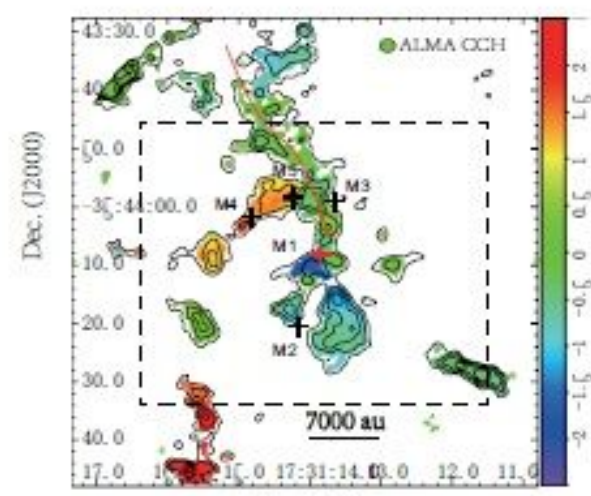

R. A. $(12000)$

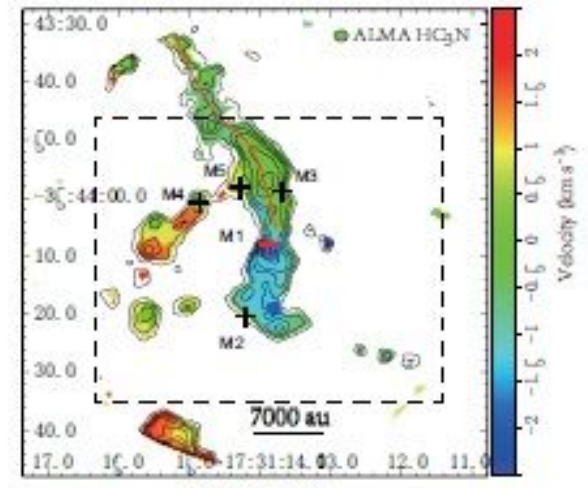

R. A. (J2000)
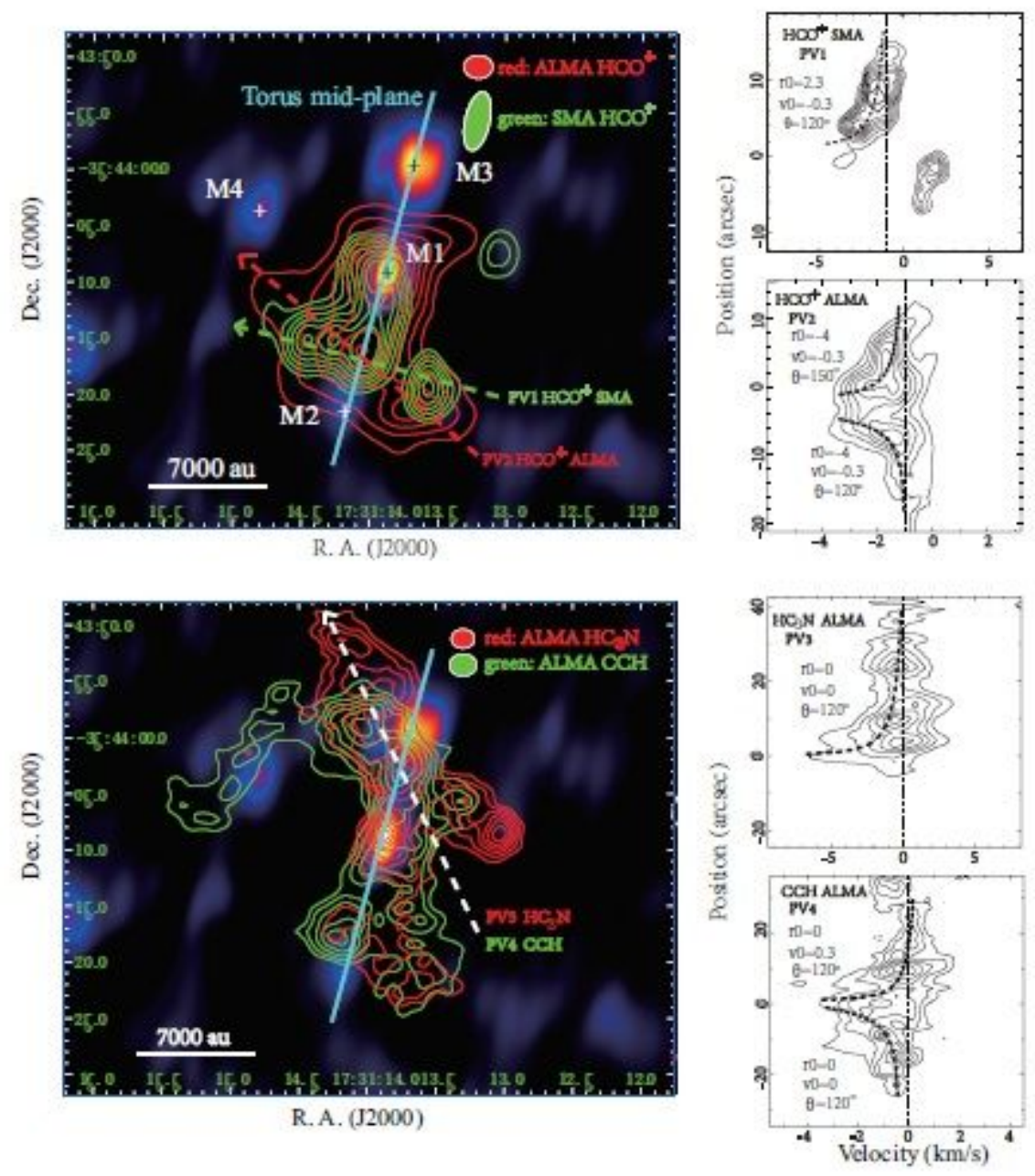


\section{Figure 4}

The gravitationally-controlled infalling streamers of chemically fresh molecules detected towards the targeted region. The upper two panels show the images of peak flux density (contours) overlaid the peak velocity (background) for the $\mathrm{CCH} \mathrm{N}=1-0, \mathrm{~J}=3=2-1=2, \mathrm{~F}=2-1$ transition (left) and the HC3N J = 11 10 transition (right) detected with ALMA. The contour levels are $(3,4,5,6) \times 0.03$ Jy beam- 1 for the CCH and $(3,4,5,6,8,10,12) \times 0.015 \mathrm{Jy}$ beam-1 for the HC3N. The red line describes the overall structures of the northeastern infalling streamers of the $\mathrm{CCH}$ and $\mathrm{HC} 3 \mathrm{~N}$. Middle and lower panels are zoomed-views of two infalling streamers towards the regions defined by the dashed-box shown in the upper panels. Middle left panel presents the southeastern infalling streamer traced by the integrated line emission from the $\mathrm{HCO}+(1-0)$ transition (red contours with levels starting from $3 \mathrm{Jy}$ beam $-1 \mathrm{~km} \mathrm{~s}-1$ in steps of $1 \mathrm{Jy}$ beam $-1 \mathrm{~km} \mathrm{~s}-1$ ) and (3-2) transition (green contours with levels starting from $0.9 \mathrm{Jy}$ beam $-1 \mathrm{~km} \mathrm{~s}-1$ in steps of 0.3 Jy beam $-1 \mathrm{~km} \mathrm{~s}-1$ ) detected with ALMA and SMA, respectively. Lower left panel shows the northwestern infalling streamer traced by the integrated line emission from the $\mathrm{CCH}$ and $\mathrm{HC} 3 \mathrm{~N}$ detected with ALMA. The contour levels for both $\mathrm{CCH}$ and $\mathrm{HC} 3 \mathrm{~N}$ start from 0.24 Jy beam $-1 \mathrm{~km} \mathrm{~s}-1$ in steps of $0.08 \mathrm{Jy}$ beam $-1 \mathrm{~km} \mathrm{~s}-1$. The background for the two panels is the $1.1 \mathrm{~mm}$ dust emission detected with SMA. The beam shapes of the two observations are given by the ellipses. The right four panels show the P-V diagrams for the directions labelled in the left two panels. The contour levels of the P-V diagrams start from $30 \%$ in steps of $10 \%$ of their peaks ( $5.36 \mathrm{Jy}$ beam -1 for the SMA HCO+; 0.89 Jy beam- 1 for the ALMA HCO+; 0.12 Jy beam-1 for the ALMA HC3N; and 0.17 Jy beam -1 for the ALMA CCH). The best free-fall models are shown by dashed curves with the fitted parameters in the corresponding P-V diagrams (see Method).

\section{Supplementary Files}

This is a list of supplementary files associated with this preprint. Click to download.

- extendedfig1.pdf

- extendedfig2.pdf

- extendedfig3.pdf 